\title{
Open Source Development with a Commercial Complementary Product or Service ${ }^{1}$
}

\author{
Ernan Haruvy, Suresh Sethi, Jing Zhou
}

University of Texas at Dallas

\begin{abstract}
Opening the source code to a software product often implies that consumers would not pay for the software product itself. However, revenues may be generated from complementary products. A software firm may be willing to open the source code to its software if it stands to build a network for its complementary products. The rapid network growth is doubly crucial in open source development, where the users of the firm's products are also contributors of code that translates to future quality improvements. To determine whether or not to open the source, a software firm must jointly optimize prices for its various products while simultaneously managing its product quality, network size, and employment strategy. Whether or not potential gains in product quality, network size, and labor savings are sufficient to justify opening the source code depends on product and demand characteristics of both the software and the complementary product as well as on the cost and productivity of in-house developers relative to open source contributors. This paper investigates these crucial elements to allow firms to reach the optimal decision in choosing between the open and closed source models.
\end{abstract}

Key words: Pricing Research; Optimal control; Open Source; Network Externalities

\footnotetext{
${ }^{1}$ We are grateful for insight and advice from Sujoy Chakravarty, Andy Novak, Ashutosh Prasad, Fang Wu, and University of Texas at Dallas seminar participants.

Corresponding author: Ernan Haruvy, eharuvy@utdallas.edu, School of Management, JO 5.1., The University of Texas at Dallas, PO Box 830688, Richardson, Texas 75083-0688, Phone: 972-883-4865, Fax: 972-883-6727.
} 


\section{Open Source Development with a Commercial Complementary Product or Service}

\section{Introduction}

The open source development paradigm has proven very successful in recent years in meeting a wide variety of software needs. Open source projects such as Linux, Apache, and Perl have become enormously successful, trampling bigger and better endowed closed source competitors. Their well publicized success has inspired tens of thousands of open source programmers worldwide and has given a boost to thousands of smaller open source projects (Schiff, 2002). In open source development, volunteersmost often users of the software- collaborate on the development of the software by contributing code and bug reports and by sharing and disseminating the code. Advantages resulting from this form of development include savings on labor costs, increased creativity associated with freedom from company restrictions, and greater development speed arising from rapid dissemination and higher relevance to end user needs (Raymond, 2001). The major caveat is that the code is freely distributed, making it difficult to sell that very same code. Given this characteristic of open source development, a firm wishing to pursue this development model must first carefully formulate its revenue model for the project and weigh the productivity benefits and labor savings against the loss of potential revenue for its software product.

Open source program development may not be appropriate for every firm. The decision to embrace this paradigm and the formulation of related strategies such as price, quality, and hiring critically depend on the business model that is used by the firm to generate revenues for its products. Given that the open source code is free, a firm which coordinates and invests its own resources in an open source project generally has a commercially sold product that would benefit from the open source code ${ }^{2}$ - by either nesting the code within its own commercially sold product, or by exploiting the complementarities that are present between the code and one of its commercially sold products. Haruvy, Prasad and Sethi (2003) evaluate the former model- where the open

\footnotetext{
${ }^{2}$ Although we cannot rule out altruism and social considerations on the part of firms and many firms claim to be motivated by such considerations, we feel it is safe to assume a profit motive. The public relations aspect of open source sponsorship may be another motive, and the modeling of such benefit is outside the scope of the present work.
} 
source code is sold as part of a commercial product. In that scenario, open source code may become a component of closed source code and the software can be made commercial. This can be done while keeping the source open by providing packaging, distribution, service or brand name or by closing the source, such as Microsoft's use of the BSD code, or the BSDi operating system (derived from Unix BSD).

In this work we examine the latter model in a monopoly setting, where the open source code is free but complements another product that is sold commercially. The open source business models discussed in the literature (Raymond, 2001; Schiff, 2002) suggest several variations on this concept: The Loss-Leader Model uses the open source code to establish brand name and lead users to the firm's other products. Netscape's Mozilla web browser is an example for such a practice. Netscape's early business model relied upon the sale of server software. The Compatible Hardware model has an open source software compatible with the firm's commercially sold hardware. An example is the AppleMaxOS $\mathrm{X}$ software which is compatible with Apple's hardware. The Service and Support model, of which Red Hat is a prominent example, provides service and support for fee to complement the open source code. Sun's Star Office falls under the Brand Compatibility model wherein the open source software complements the firm's product lines and brand image. Finally, under the Information model a firm charges for information associated with the open source code. Consulting services often charge for information relating to open source documentation, maintenance, etc. Notice that the models discussed here can also apply to a freeware software product - a software product which is free but whose code is not necessarily open to the public. However, the key difference lies in the development efforts which are less expensive and more rapid in the open source model. For a discussion of when the freeware model would be most appropriate see Haruvy and Prasad (1998; 2001). 
Table 1. Open source Business Models

\begin{tabular}{|c|c|}
\hline Business Model & Example \\
\hline Loss-leader & Netscape's Mozilla \\
\hline Compatible Hardware & Apple MaxOS X \\
\hline Service and Support & Red Hat \\
\hline Brand Compatibility & Sun's StarOffice \\
\hline Information & $\begin{array}{c}\text { Wide variety of vendors, } \\
\text { including IBM, Oracle, and } \\
\text { consulting firms }\end{array}$ \\
\hline
\end{tabular}

Note that an open source product in the categories discussed here need not be a full software product. It can be a more limited group of modules or classes for existing open source software. For example, IBM initiates and supports various Apache-related projects and releases related code even when it is developed in-house. IBM's main source of revenue in this case is derived from selling web servers, which benefit greatly from improvements and additions to Apache.

Given the known business models, not every firm should pursue open source as its software model. When a firm decides to pursue a software project, it will generally have an in-house developed prototype and some assessment of the in-house development potential. If the potential contribution from outside programmers is not perceived to be large, obviously the firm should not pursue open source. Furthermore, when the benefit to the firm's complementary commercial product is small relative to the value of the inhouse developed software to the consumers, the firm should keep the software product proprietary and extract as much of the consumer surplus as it can.

Our analysis is intended to uncover the relationships between important product characteristics and the projected success of open and closed source initiatives. Our main findings are that firms should pursue open source development, as opposed to closed source development, if (1) user contributions (or programmers who are users) are critical to development: e.g., bug reports specific to the code, (2) the relative effectiveness of closed source programmers is low, (3) in-house production is expensive, or (4) initial quality is low. In addition, other important insights are revealed: (5) in finite horizon 
settings, software quality decreases towards the end of the product's life in the closed source but not in the open source. This finding has important welfare implications. (6) When initial quality of the complementary product is high, the closed source software product may be priced at zero initially for a long period of time, making it freeware. In such a case, open source will dominate the closed source.

The solution approach taken here is optimal control. This approach is ideal here since the firm is able to vary the price of its commercial products from period to period and since related variables (state variables) such as quality and network size vary over time. Such approach has been often taken in the literature to address dynamic pricing problems (e.g., Chintagunta and Rao, 1996), dynamic quality problems (Muller and Peles, 1988), and dynamic advertising expenditures problems (for a survey of that literature see Feichtinger, Hartl and Sethi, 1994). The approach presented here allows for dynamic pricing in the presence of network externalities (e.g., Dhebar and Oren, 1985) as well as for dynamic quality, where the former is a control variable and the latter is a state variable.

Also note the parallel between the present paper and some works in the innovation diffusion literature. Diffusion is generally defined as the process by which information about an innovation is communicated through a social system (Rogers, 1983). In essence, diffusion can be thought of a special type of network effect ${ }^{3}$ which, when accounting for the dependence between demand and price, translates to a dynamic pricing problem for a product (e.g., Kalish, 1983; Kalish and Lilien, 1983; Nascimento and Vanhonacker, 1988).

The approach we take here expands on the above dynamic pricing literature by examining a scenario of two complementary goods, one of which can be developed through user contributions contingent on it being free. The paper is organized as follows: Section 2 lays out the basic model and assumptions. Section 3 develops the theory and its analytical implications as they relate to dynamics and equilibrium in both finite and

\footnotetext{
${ }^{3}$ A network effect implies that the larger the network of existing users the more likely non-adopters are to adopt. In diffusion models, this is generally captured by the 'coefficient of imitation' (Bass, 1969). At the individual level, diffusion can also be explained by decreased uncertainty and risk on various attributes as the network increases (Oren and Schwartz, 1988; Chaterjee and Eliashberg, 1990; Lattin and Roberts, 1989; also see Mahajan, Muller, and Bass, 1990, for a comprehensive survey).
} 
infinite horizon settings. Section 4 pursues numerical simulations for comparative statics and further insights. Section 5 concludes.

\section{The models and setting}

The firm has two products, product 1 and product 2 , which have different interdependent demands, $D_{1}$ and $D_{2}$, respectively. In the case of closed source development, all quality improvements arise in-house as a function of the number of inhouse developers. In the case of open source development, all quality improvements come from the users, as a function of the size $m$ of the network.

$Q_{1}(t)=$ The quality of the software at time $t$.

$m(t)=$ size of network of users (i.e., installed base) at time $t$.

$N(t)=$ Number of in-house developers at time $t$.

The model is as follows: The profit in a given period is the revenue $P_{1} D_{1}$ from the software, plus the revenue $P_{2} D_{2}$ from the complementary product, minus development costs if applicable. In the open source case, $P_{1}$ is by definition zero, and so the revenue is only $P_{2} D_{2}$. This loss of revenue may be offset by the fact that development costs are zero. In the closed source case, development costs increase in the number of in-house developers, $N$. We assume a cost function of $w N^{2}$ in accordance with the economic principle of increasing marginal cost ${ }^{4}$. In the closed source case, the in-house contributors improve the quality of the software over time. That is,

$$
\dot{Q}_{1}=k N-\delta Q_{1}, Q_{1}(0)=Q_{0}>0,
$$

The parameter $k$ denotes the productivity or effectiveness of the in-house closed source programmers. When $k$ is high, closed source programmers are very effective and open

\footnotetext{
${ }^{4} w$ is not unit wage but rather a cost parameter. One can think of a generalized cost function $w N^{\gamma}$. Given the law of increasing marginal costs, $\gamma=2$ is a reasonable example.
} 
source development may not be warranted. It is assumed that the quality depreciates over time at a constant proportional rate $^{5} \delta>0$. This depreciation can be thought of as obsolescence relative to exogenous technological progress. It is assumed that initial quality of the software is positive. No project starts with zero quality. At the very least, an idea or concept exists, which can be quantified as miniscule quality. Since the source is closed to the public, contributions by users are not possible. In the open source case, on the other hand, there are no in-house developers and all contribution is through users. Hence,

$$
\dot{Q}_{1}=\alpha m-\delta Q_{1}, Q_{1}(0)=Q_{0}>0
$$

The parameter $\alpha$ represents the level of involvement by the open source user community, which includes users of both the software and of the complementary product. The size $m$ of the network of users increases each period by the number of new users of both products. However, the users of both products need not be equally weighted. A user of the software may be more or less valuable to the network than a user of the complementary product. The parameter $a>0$ measures this relative weight. We also assume separability between demands for the two products in the network, though the two are complements. That is, a user who uses both products has a higher weight in the network than a user who uses only one product. The parameter $\varepsilon>0$ is the rate of depreciation of the network or the rate of exit. Hence,

$$
\dot{m}=a D_{1}+D_{2}-\varepsilon m, \quad m(0)=m_{0}>0
$$

The demand of the complementary product $D_{2}$ decreases as the price of the complementary product $P_{2}$ increases, and increases as the size $m$ of the network of users

\footnotetext{
${ }^{5}$ Alternatively, we could have a constant depreciation each period. That could result in explosive solutions $\left(Q_{1} \rightarrow 0\right.$ ) and no steady state. It is reasonable to assume that higher quality would manifest in more rapid obsolescence relative to progressing technologies.
} 
and the quality of the complementary product $Q_{2}$ increase. $Q_{2}$ is assumed to be positive and constant. We assume the demand function of the complementary product as follows:

$$
D_{2}=\exp \left(-\frac{P_{2}}{m Q_{2}}\right)
$$

We use the exponential demand function as in Haruvy, Prasad, and Sethi (2003). Such a function exhibits the desired properties of a demand function in price, quality, and network size. This functional form has the additional advantage that it precludes the possibility of negative demand.

The demand of the software $D_{1}$ decreases as the price of the software $P_{1}$ increases, and increases as the quality of the software $Q_{1}$ increases. We assume the demand of the software to be as follows:

$$
D_{1}=D_{2} \exp \left(-\frac{P_{1}+c}{Q_{1}}\right)
$$

The multiplication of the exponential by $D_{2}$ represents the effect of complementarity. That is, the demand for the software product increases with the demand for the complementary product. The positive constant $\mathrm{c}$ in equation (5) is necessary to keep the numerator strictly above zero in the open source case. Without the positive constant c, the demand would cease to be a function of $Q_{1}$ in the open source case. Since the price $P_{1}$ for the open source software product is zero, in the open source case we have:

$$
D_{1}=D_{2} \exp \left(-\frac{c}{Q_{1}}\right)
$$

We summarize the two models as follows: 
Table 2. The models

\begin{tabular}{|ll|ll|}
\hline \multicolumn{1}{|l|}{ Closed Source } & \multicolumn{1}{l|}{ Open Source } \\
\hline Max $\int_{0}^{\infty} e^{-\rho t}\left(P_{1} D_{1}+P_{2} D_{2}-w N^{2}\right) d t$ & $\operatorname{Max} \int_{0}^{\infty} e^{-\rho t}\left(P_{2} D_{2}\right) d t$ & \\
s.t. & s.t. & \\
& $\dot{Q}_{1}=k N-\delta Q_{1}$, & $\dot{Q}_{1}=\alpha m-\delta Q_{1}$, & $Q_{1}(0)=Q_{0}$ \\
$\dot{m}=a D_{1}+D_{2}-\varepsilon m$, & $m(0)=m_{0}$ & $\dot{m}=a D_{1}+D_{2}-\varepsilon m$, & $m(0)=m_{0}$ \\
where & where & \\
$\qquad D_{1}=D_{2} \exp \left(-\frac{P_{1}+c}{Q_{1}}\right)$ & $D_{1}=D_{2} \exp \left(-\frac{c}{Q_{1}}\right)$ & \\
$D_{2}=\exp \left(-\frac{P_{2}}{m Q_{2}}\right)$ & $D_{2}=\exp \left(-\frac{P_{2}}{m Q_{2}}\right)$
\end{tabular}

At this point some more discussion is warranted on the relationship between $D_{l}$ (demand for software) and $D_{2}$ (demand for the complement). $D_{1}$ and $D_{2}$ affect each other, but in different ways. $D_{1}$ is affected by $D_{2}$ directly, through complementarities. That is, a portion of the complement's users $\left(D_{2}\right)$ users may wish to use the software $\left(D_{1}\right)$. However, the software users can use the software independently of the complementary good (they could substitute any number of other products). $D_{2}$ is affected by $D_{1}$ indirectly, through the network size (which is a function of purchases of both 1 and 2). $D_{2}$ is affected by the network because of network externalities. That is, it is affected directly by the size of the network. People derive utilities from other people using the product, though newsgroups, file sharing, increased service and compatible goods. $D_{l}$ is not affected by the network directly. However, in the open source case, D1 is affected by the network indirectly through the impact of the network on the size of the code, $Q_{1}$.

\section{Analytical investigation}

\subsection{Open Source}


The firm maximizes its discounted (discount rate is $\rho>0$ ) profit stream, which consists of the price for the commercial product (the price for the open source software product, $P_{1}$, is zero) multiplied by the demand for the commercial product at each period.

$$
\operatorname{Max} \int_{0}^{\infty} \exp (-\rho t) P_{2} \exp \left(-\frac{P_{2}}{m Q_{2}}\right) d t
$$

s.t.

$$
\begin{array}{ll}
\dot{Q}_{1}=\alpha m-\delta Q_{1}, & Q_{1}(0)=Q_{0}, \\
\dot{m}=a \exp \left(-\frac{c}{Q_{1}}\right) \exp \left(-\frac{P_{2}}{m Q_{2}}\right)+\exp \left(-\frac{P_{2}}{m Q_{2}}\right)-\varepsilon m, & m(0)=m_{0}, \\
\left.P_{2} \geq 0^{6} \quad \text { (The price cannot be negative }\right) &
\end{array}
$$

We form the Hamiltonian:

$$
\begin{aligned}
L=P_{2} \exp ( & \left.-\frac{P_{2}}{m Q_{2}}\right)+\lambda\left(\alpha m-\delta Q_{1}\right) \\
+\mu\left[a \exp \left(-\frac{c}{Q_{1}}\right) \exp \left(-\frac{P_{2}}{m Q_{2}}\right)+\exp \left(-\frac{P_{2}}{m Q_{2}}\right)-\varepsilon m\right]+\eta_{2} P_{2} & ,
\end{aligned}
$$

From this we get the adjoint equations

$$
\begin{aligned}
& \dot{\lambda}=\rho \lambda-\frac{\partial L}{\partial Q_{1}}=(\rho+\delta) \lambda-\frac{a c \mu}{Q_{1}^{2}} \exp \left(-\frac{c}{Q_{1}}\right) \exp \left(-\frac{P_{2}}{m Q_{2}}\right), \\
& \dot{\mu}=\rho \mu-\frac{\partial L}{\partial m}=(\rho+\varepsilon) \mu-\alpha \lambda-\frac{P_{2}}{m} \exp \left(-\frac{P_{2}}{m Q_{2}}\right)\left[\frac{P_{2}}{m Q_{2}}+\frac{\mu}{m Q_{2}}\left(a \exp \left(-\frac{c}{Q_{1}}\right)+1\right)\right],
\end{aligned}
$$

The optimal control must satisfy

$$
\frac{\partial L}{\partial P_{2}}=\left[1-\frac{P_{2}}{m Q_{2}}-\frac{\mu}{m Q_{2}}\left(a \exp \left(-\frac{c}{Q_{1}}\right)+1\right)\right] \exp \left(-\frac{P_{2}}{m Q_{2}}\right)+\eta_{2}=0,
$$

and the Hamiltonian multiplier $\eta_{2}$ must satisfy the complementary slackness condition

\footnotetext{
${ }^{6}$ We assume the lower bound of price is zero. It is possible to have a positive price lower bound.
} 


$$
\eta_{2} \geq 0, \quad \eta_{2} P_{2}=0
$$

From (11) and (12), we can have:

$$
P_{2}=\left\{\begin{array}{cl}
m Q_{2}-\mu\left(a \exp \left(-\frac{c}{Q_{1}}\right)+1\right), & m Q_{2}-\mu\left(a \exp \left(-\frac{c}{Q_{1}}\right)+1\right) \geq 0 \\
0, & m Q_{2}-\mu\left(a \exp \left(-\frac{c}{Q_{1}}\right)+1\right)<0
\end{array}\right.
$$

From 13, we get an equation of motion for $P_{2}$.

$$
\dot{P}_{2}=\left\{\begin{array}{cl}
\dot{m} Q_{2}-\dot{\mu}\left(a e^{-c / Q_{1}}+1\right)-\mu \dot{Q}_{1}\left(\frac{a c}{Q_{1}^{2}} e^{-c / Q_{1}}\right), & m Q_{2}-\mu\left(a \exp \left(-\frac{c}{Q_{1}}\right)+1\right) \geq 0 \\
0, & m Q_{2}-\mu\left(a \exp \left(-\frac{c}{Q_{1}}\right)+1\right)<0
\end{array}\right.
$$

Now the following question arises: In the formulation with infinite horizon, does the optimal solution converge to a steady state as time approaches infinity? The practicality of such a concern is somewhat uncertain. After all, neither software product nor company can last forever. Nevertheless, the convergence question is central to dynamic problems since the convergence property is suggestive of the solution to the finite horizon problem.

Since we want to maximize the present value of the revenue streams, the price $P_{2}$ must be positive in steady state for any viable firm. Therefore, the steady state value of the Hamiltonian multiplier is zero, i.e., $\bar{\eta}_{2}=0$.

We have the following five equations in the steady state: 


$$
\begin{aligned}
& \dot{Q}_{1}=\alpha m-\delta Q_{1}=0 \\
& \dot{m}=a \exp \left(-\frac{c}{Q_{1}}\right) \exp \left(-\frac{P_{2}}{m Q_{2}}\right)+\exp \left(-\frac{P_{2}}{m Q_{2}}\right)-\varepsilon m=0 \\
& \dot{\lambda}=(\rho+\delta) \lambda-\frac{a c \mu}{Q_{1}^{2}} \exp \left(-\frac{c}{Q_{1}}\right) \exp \left(-\frac{P_{2}}{m Q_{2}}\right)=0 \\
& \dot{\mu}=(\rho+\varepsilon) \mu-\alpha \lambda-\frac{P_{2}}{m} \exp \left(-\frac{P_{2}}{m Q_{2}}\right)\left[\frac{P_{2}}{m Q_{2}}+\frac{\mu}{m Q_{2}}\left(a \exp \left(-\frac{c}{Q_{1}}\right)+1\right)\right]=0 \\
& P_{2}=m Q_{2}-\mu\left(a \exp \left(-\frac{c}{Q_{1}}\right)+1\right)
\end{aligned}
$$

Though a closed form analytical solution is intractable, it is of importance to determine whether a unique solution exists to this system of equations.

Proposition 1: In the open source case:

(a) The marginal benefit $\mu$ of increasing the size of the network is positive.

(b) The marginal benefit $\lambda$ of increasing the software quality is positive.

(c) The optimal profit increases with $\alpha$.

Proof: See Appendix A for detail.

Corollary 1.1: In the open source model, $m Q_{2}$ is an upper bound to $P_{2}$.

Proof: The proof immediately follows from Proposition 1. Specifically, since $\mu$ is always positive and the parameter $a$ is positive, by equation (19), $P_{2}$ is less than or equal to $m Q_{2}$.

Proposition 2: There exists a solution to the open source model's system of steady state equations. That is, there exists a long term stationary equilibrium $\bar{Q}_{1}, \bar{m}, \bar{P}, \bar{\lambda}, \bar{\mu}$, and $\bar{\eta}_{2}=0$.

Proof: See Appendix B for detail. 
Proposition 3: The solution to the open source model's system of steady state equations is unique. That is, there exists a unique $\bar{Q}_{1}, \bar{m}, \bar{P}_{2}, \bar{\lambda}, \bar{\mu}$, and $\bar{\eta}_{2}=0$.

Proof: See Appendix C for detail.

\subsection{Closed Source}

The firm maximizes its discounted profit stream, which is the revenue of the software and the commercial product minus the development costs at each period.

$$
\operatorname{Max} \int_{0}^{\infty} \exp (-\rho t)\left[P_{1} \exp \left(-\frac{P_{1}+c}{Q_{1}}\right) \exp \left(-\frac{P_{2}}{m Q_{2}}\right)+P_{2} \exp \left(-\frac{P_{2}}{m Q_{2}}\right)-w N^{2}\right] d t
$$

s.t.

$$
\begin{array}{cc}
\dot{Q}_{1}=k N-\delta Q_{1} & Q_{1}(0)=Q_{0} \\
\dot{m}=a \exp \left(-\frac{P_{1}+c}{Q_{1}}\right) \exp \left(-\frac{P_{2}}{m Q_{2}}\right)+\exp \left(-\frac{P_{2}}{m Q_{2}}\right)-\varepsilon m & m(0)=m_{0} \\
P_{1}, P_{2} \geq 0^{7}, N \geq 0 \text { (The prices and the number of in-house developers is } \\
\text { nonnegative) }
\end{array}
$$

We form the Hamiltonian:

$$
\begin{aligned}
L= & P_{1} \exp \left(-\frac{P_{1}+c}{Q_{1}}\right) \exp \left(-\frac{P_{2}}{m Q_{2}}\right)+P_{2} \exp \left(-\frac{P_{2}}{m Q_{2}}\right)-w N^{2}+\lambda\left(k N-\delta Q_{1}\right) \\
& +\mu\left[a \exp \left(-\frac{P_{1}+c}{Q_{1}}\right) \exp \left(-\frac{P_{2}}{m Q_{2}}\right)+\exp \left(-\frac{P_{2}}{m Q_{2}}\right)-\varepsilon m\right]+\eta_{1} P_{1}+\eta_{2} P_{2}+\eta_{3} N
\end{aligned}
$$

From this we get the adjoint equations

$$
\begin{aligned}
& \dot{\lambda}=(\rho+\delta) \lambda-\frac{\left(P_{1}+a \mu\right)\left(P_{1}+c\right)}{Q_{1}^{2}} \exp \left(-\frac{P_{1}+c}{Q_{1}}\right) \exp \left(-\frac{P_{2}}{m Q_{2}}\right) \\
& \dot{\mu}=(\rho+\varepsilon) \mu-\frac{P_{2}}{m^{2} Q_{2}} \exp \left(-\frac{P_{2}}{m Q_{2}}\right)\left[\left(P_{1}+a \mu\right) \exp \left(-\frac{P_{1}+c}{Q_{1}}\right)+P_{2}+\mu\right]
\end{aligned}
$$

\footnotetext{
${ }^{7}$ We assume the lower bound of price is zero in order to have one less parameter in the model. A positive price lower bound can easily be incorporated.
} 
The optimal control must satisfy

$$
\begin{aligned}
& \frac{\partial L}{\partial P_{1}}=\left[1-\frac{P_{1}+a \mu}{Q_{1}}\right] \exp \left(-\frac{P_{1}+c}{Q_{1}}\right) \exp \left(-\frac{P_{2}}{m Q_{2}}\right)+\eta_{1}=0 \\
& \frac{\partial L}{\partial P_{2}}=\left[1-\frac{P_{2}}{m Q_{2}}-\frac{\mu}{m Q_{2}}-\frac{P_{1}+a \mu}{m Q_{2}} \exp \left(-\frac{P_{1}+c}{Q_{1}}\right)\right] \exp \left(-\frac{P_{2}}{m Q_{2}}\right)+\eta_{2}=0 \\
& \frac{\partial L}{\partial N}=-2 w N+k \lambda+\eta_{3}=0
\end{aligned}
$$

and the Hamiltonian multipliers $\eta_{1}, \eta_{2}$ and $\eta_{3}$ must satisfy the complementary slackness condition

$$
\begin{array}{ll}
\eta_{1} \geq 0, & \eta_{1} P_{1} \geq 0 \\
\eta_{2} \geq 0, & \eta_{2} P_{2} \geq 0 \\
\eta_{3} \geq 0, & \eta_{3} N \geq 0
\end{array}
$$

From (24) -- (27), we can have:

$$
\begin{aligned}
& P_{1}=\left\{\begin{array}{cc}
Q_{1}-a \mu, & Q_{1}-a \mu \geq 0 \\
0, & Q_{1}-a \mu<0
\end{array}\right. \\
& P_{2}=\left\{\begin{array}{cc}
m Q_{2}-\mu-\left(P_{1}+a \mu\right) \exp \left(-\frac{P_{1}+c}{Q_{1}}\right), & m Q_{2}-\mu-\left(P_{1}+a \mu\right) \exp \left(-\frac{P_{1}+c}{Q_{1}}\right) \geq 0 \\
0, & m Q_{2}-\mu-\left(P_{1}+a \mu\right) \exp \left(-\frac{P_{1}+c}{Q_{1}}\right)<0
\end{array}\right. \\
& N=\left\{\begin{array}{cl}
\frac{k \lambda}{2 w}, & \frac{k \lambda}{2 w} \geq 0 \\
0, & \frac{k \lambda}{2 w}<0
\end{array}\right.
\end{aligned}
$$

Since we wish to maximize the present value of the net profit stream, prices $P_{1}$ and $P_{2}$ must be positive in steady state for any viable firm. Therefore, the steady state values of the Hamiltonian multipliers of prices are zero, i.e., $\bar{\eta}_{1}=\bar{\eta}_{2}=0$. In addition, the equation of motion $\dot{Q}_{1}=k N-\delta Q_{1}=0$ implies that the steady state value of the number 
of in-house programmers, $\bar{N}$, is positive. Therefore, the steady state value of the Hamiltonian multiplier of the number of in-house programmers must be zero, i.e., $\bar{\eta}_{3}=0$. The following seven equations must hold in steady state:

Table 4 Steady State equations for the closed source model

$$
\begin{aligned}
& \dot{Q}_{1}=k N-\delta Q_{1}=0 \\
& \dot{m}=a \exp \left(-\frac{P_{1}+c}{Q_{1}}\right) \exp \left(-\frac{P_{2}}{m Q_{2}}\right)+\exp \left(-\frac{P_{2}}{m Q_{2}}\right)-\varepsilon m=0 \\
& \dot{\lambda}=(\rho+\delta) \lambda-\frac{\left(P_{1}+a \mu\right)\left(P_{1}+c\right)}{Q_{1}^{2}} \exp \left(-\frac{P_{1}+c}{Q_{1}}\right) \exp \left(-\frac{P_{2}}{m Q_{2}}\right)=0 \\
& \dot{\mu}=(\rho+\varepsilon) \mu-\frac{P_{2}}{m^{2} Q_{2}} \exp \left(-\frac{P_{2}}{m Q_{2}}\right)\left[\left(P_{1}+a \mu\right) \exp \left(-\frac{P_{1}+c}{Q_{1}}\right)+P_{2}+\mu\right]=0 \\
& P_{1}=Q_{1}-a \mu \\
& P_{2}=m Q_{2}-\mu-\left(P_{1}+a \mu\right) \exp \left(-\frac{P_{1}+c}{Q_{1}}\right) \\
& N=\frac{k \lambda}{2 w}
\end{aligned}
$$

Proposition 4: In the closed source case:

(a) The marginal benefit $\mu$ of increasing the size of the network is positive.

(b) The marginal benefit $\lambda$ of increasing the software quality is positive.

(c) The optimal profit increases in $k$ and decreases in $w$.

Proof: See Appendix D for detail.

Corollary 4.1: In the closed source model, $m Q_{2}$ is an upper bound to $P_{2}$ and $Q_{1}$ is an upper bound to $P_{1}$.

Proof: The proof immediately follows from Proposition 4. Specifically, since $\mu$ is always positive and the parameter $a$ is positive, by equation (35), $P_{1}$ is less than or equal to $Q_{1}$. By equation (36) $P_{2}$ is less than or equal to $m Q_{2}$. 
Proposition 5: There exists a solution to the closed source model's system of steady state equations. That is, there exists $\bar{Q}_{1}, \bar{m}, \bar{P}_{1}, \bar{P}_{2}, \bar{N}, \bar{\lambda}$ and $\bar{\mu}$.

Proof: See Appendix E for detail.

Proposition 6: The solution to the closed source model's system of steady state equations is unique. That is, there exists a single $\overline{Q_{1}}, \bar{m}, \bar{P}_{1}, \overline{P_{2}}, \bar{N}, \bar{\lambda}$ and $\bar{\mu}$.

Proof: See Appendix F for detail.

\section{Finite horizon ( $T$ ) with no salvage value}

Suppose the products, both the software and the complementary product, have finite lives with a known terminal period. That could be due to an anticipated release of a new generation of products or technologies or due to a known date for the firm to cease operations. In that case, we can set the salvage value to zero. When the time horizon is sufficiently long and the discounting significant, profit comparisons in the finite and infinite horizons should be close. The formulation of the finite horizon problem with zero salvage value is the same as before, but with the addition of the boundaries $\lambda(T)=0$ and $\mu(T)=0$.

Proposition 7: In the closed source model, the number of in-house developers $N$ declines over time for a finite horizon problem with zero salvage value and eventually $N$ goes to zero at $T$. On the other hand, $N$ approaches steady state over time in the infinite horizon problem.

Proof: See Appendix G for detail and Figure 2 for numerical results.

\section{Numerical Analysis}

We examine different scenarios numerically. In both open source and closed source models, and both finite horizon without salvage value and infinite horizon settings. We use Fortran code and Excel spreadsheets to find numerical solutions. Due to the computational limitations, numerical solutions in both the finite and infinite 
horizon settings are the result of approximations (e.g., Sethi and Thompson, 2000, pp. 4850). We use the following parameters:

$$
\delta=0.03, \varepsilon=0.01, \rho=0.1, a=c=k=w=1, \alpha=1, Q_{2}=1
$$

Due to network externalities, prices for both products in the closed source model and the price for the complementary product in the open source model begin low in order to quickly establish the network's installed base and gradually increase as the network size increases, as figure 1 demonstrates.

\section{$<$ Insert Figure 1 here $>$}

We can see that price in the initial periods, for both software and complementary product is zero. This is because of the network externality effect. In order to build the network and extract the maximal surplus, the producer is willing to sacrifice short-term profits for larger future profits.

In the closed source model, hiring is massive early on due to the need to rapidly increase quality in order to build up the network As a result, we see quality increases rapidly in the initial time period. In finite horizon problem, hiring eases up over time and quality begins decreasing towards the end of the life of the products. This is quite intuitive since given an anticipated product cease, it is no use to hire people and improve the quality at the end of the life of the product. In the infinite horizon problem, hiring approaches steady state over time and so does the quality. In the open source model, quality increases continuously since in this scenario quality increases as the size of network of users increases. The difference between finite and infinite horizon problems is that quality goes to steady state in infinite horizon problem and it is not the case in finite horizon problem, as figure 2 demonstrates.

$<$ Insert Figure 2 here $>$ 
An important difference to note between the open source and closed source in the finite horizon setting is that in the closed source case software quality decreases towards the end of the life of the software, whereas in the open source case it continues to increase. This will always be universally true and not just in the numerical example shown here. This is because the firm will not find it optimal to invest in quality when the product nears its death, whereas the open source community, not motivated by profit, will continue to improve. This presents interesting welfare implications to open source development, particularly if the benefit to society from increased quality is substantial.

Next, we illustrate the idea that when a firm has to choose between open source and closed source, that choice may depend on the values of the parameters $\alpha, k, w$, and the initial quality $Q_{0}$. From proposition 1 and proposition 4 it is clear that threshold values exist for the parameters $\alpha, k, w$, above which one model dominates and below which the other model dominates.

The numerical analysis clearly confirms that thresholds exist. There appears to exist a threshold open source community involvement, $\bar{\alpha}$, above which open source will be preferred to closed source. (See Figure 3)

\section{$<$ Insert Figure 3 here $>$}

Similarly, there seems to exist a threshold in-house programmer productivity, $\bar{k}$, above which closed source will be preferred to open source. (See Figure 4)

\section{$<$ Insert Figure 4 here $>$}

In considering whether to develop in-house software, the firm must consider wages as well as in-house productivity. As for in-house productivity, we see a threshold wage factor, $\bar{w}$, below which open source will be preferred to closed source. (See Figure 5)

\section{$<$ Insert Figure 5 here $>$}


Finally, initial quality is a critical consideration. When initial quality is high, the firm has little to benefit from opening the source code to the open source community. As such, there exists a threshold initial quality, $\mathrm{Q}_{0}$, below which open source will be preferred to closed source. (See Figure 6)

$<$ Insert Figure 6 here $>$

In addition to comparative statics, we attempt to examine some of the corollaries numerically. First, we attempt to numerically verify corollary 1.1. According to that result, in the open source model, $m Q_{2}$ is an upper bound to $P_{2}$. Figure 7 suggests that $m Q_{2}$ is not only an upper bound on $P_{2}$ but that $P_{2}$ closely tracks $m Q_{2}$ in magnitude and slope.

\section{$<$ Insert Figure 7 here $>$}

Next, we illustrate corollary 4.1 numerically. Specifically, we wish to verify that in the closed source model, $m Q_{2}$ is an upper bound to $P_{2}$ and $Q_{1}$ is an upper bound to $P_{1}$, and to see how closely prices track their upper bound. Surprisingly, while $P_{1}$ closely tracks quality in slope and magnitude, $P_{2}$ slowly diverges from $m Q_{2}$.

\section{$<$ Insert Figure 8 here $>$}

Moreover, we find that in the closed source case, the higher the initial quality of the complementary product, $Q_{2}$, the longer the price of the software $P_{1}$ will remain at zero initially. In addition, the higher the $Q_{2}$, the higher the $P_{2}$ and the lower the $P_{1}$ at the end of the time horizon. 


\section{Conclusions}

Whereas the speed and creativity associated with open source development are well accepted, the profit potential is not always recognized. As companies become increasingly involved in open source projects, the profit implications of open source for companies must be better understood. In this article we argue that the profitability of open source software may come from a product which is a complement to the open source code.

A firm considering open source development as an alternative to closed source development would need to carefully review the relationship between the software in question and the firm's other products. If the software is found to enhance the usefulness or quality of complementary products and/or if the users of the software and the users of the complementary product belong to the same network, the complementarity can be exploited. By dynamically and simultaneously managing price, product quality, network size, and hiring for both products, the firm will be able to best exploit the complementarity. This effort may or may not benefit from opening the source of the product. Without a clear model of how to exploit freeware, open source development as a substitute to in-house closed source development may be detrimental to a firm's profitability. Our analytical derivation and simulations demonstrate that under various conditions open source may not be beneficial to a firm. On the other hand, there are scenarios where the opposite is the case.

Specifically, open source community involvement is critical to the success of open source initiative. Only above a critical level of community involvement, open source becomes a viable alternative to closed source. To the extent that community involvement can be influenced by the firm's efforts or influence, such efforts must complement the pricing decisions evaluated here. In-house programmer productivity was shown to be the opposite side of the same coin. More productive and efficient in-house programmers result in less reliance on open source. However, wage is critical in that respect. If programmer productivity is high but wage is higher than some threshold, our results show that open source would be preferred. Finally, if the initial quality of the software is high, development becomes a less critical consideration and extraction of 
surplus can begin immediately. In such a case, the firm would prefer to charge a positive price for the software and close the code.

We also characterized price, quality, and hiring paths for firms under both the open source and closed source models. We find that due to network externalities, prices for both products in the closed source model and for the complementary product in the open source model will begin low in order to quickly establish the network's installed base and gradually increase as the network size increases. We find that in the closed source case, the higher the initial quality of the complementary good, the longer the price of the software will remain at zero initially. That is, when more surplus can be extracted from the complementary good, it may be optimal to have the software as a freeware for a long period of time, even when the source is closed.

In the closed source model for products with finite lives, quality will rapidly increase early on to build the network size and begin decreasing towards the end of the life of the products. Similarly, hiring will be massive early on and will ease up over time. This is due to the need to rapidly increase quality in order to establish the network. This is not the case in infinite horizon closed source model and not in the finite or infinite horizon open source models. As such, it may be argued that for finitely lived products, open source improves society's welfare in terms of both quality and productivity.

Future research should examine other forms of revenue extraction from open source as well as the problem from a social planner's perspective. We would further like to examine the effect of competitive pressures on the firm's decision to use open source. When the firm opens it source, it may implicitly provide advantages to competitors. Compatibility and legal protection will become paramount considerations when competition is considered. 


\section{References}

Bass, F. M. (1969), "A New Product Growth Model for Consumer Durables," Management Science, 15, 215-227.

Brock, W. A., and Scheinkman, J. A. (1977), "The global asymptotic stability of optimal control with applications to dynamic economic theory," Applications of Control Theory to Economic Analysis. Ed. By J.D. Pitchford and St. J. Turnovsky. Amsterdam - New York - Oxford 1997, Essay8, 173-205

Chaterjee, R. and J. Eliashberg (1990), "The Innovation Diffusion Process in a Heterogeneous Population: A Micromodeling Approach," Management Science, $36,1057-1079$.

Chintagunta, P. K., and Rao, V. R. (1996). "Pricing strategies in a dynamic duopoly: A differential game model," Management Science 42, 11, 1501-1514.

Dhebar, A. and S. Oren (1985), "Optimal Dynamic Pricing for Expanding Networks," Marketing Science, 4, 336-351.

Feichtinger, Gustav; Richard F. Hartl; Suresh P. Sethi (1994), "Dynamic Optimal Control Models in Advertising: Recent Developments," Management Science, 40 (2)., 195-226.

Haruvy, E. and Prasad, A. (1998), "Optimal Product Strategies in the Presence of Network Externalities," Information Economics and Policy 10, 489-499.

Haruvy, E. and Prasad, A. (2001), "Optimal Product Strategies in the Presence of Network Externalities: An Evolutionary Game Theoretical Approach," Journal of Evolutionary Economics 11, 231-248.

Haruvy, E., A. Prasad, and S. Sethi (2003), "Harvesting Altruism in Open Source Software Development," forthcoming in Journal of Optimization Theory and Applications.

Hsu, J. and A. Meyer (1968), Modern control principles and applications (McGraw-Hill, New York 1968)

Kalish, S. (1983), "Monopolist Pricing with Dynamic Demand and Production Cost," Marketing Science, 2, 135-159.

Kalish, S. and G. Lilien (1983), "Optimal Price Subsidy for Accelerating the Diffusion of Innovation," Marketing Science, 407-420

Lattin, J. M. and J. H. Roberts (1989), "Modeling the Role of Risk Adjusted Utility in the Diffusion of Innovations," working paper 1019, Graduate School of Business, Stanford University.

Mahajan, V., Muller, E. and F. M. Bass (1990), "New Product Diffusion Models in Marketing: A Review and Directions for Research, Journal of Marketing, 54, 126.

Muller, Eitan and Yoram Peles (1988), "The Dynamic Adjustment of Optimal Durability and Quality," International Journal of Industrial Organization, 6, pp. 499-507. 
Nascimento, F. and W. Vanhonacker (1988), "Optimal Strategic Pricing of Reproducible Consumer Products," Management Science, 34, 921-937.

Oren, Shmuel S. and Rick G. Schwartz (1988), "Diffusion of New Products in RiskSensitive Markets." Journal of Forecasting 7, 273-287.

Raymond, E. S. (2001), The Cathedral and the Bazaar: Musings on Linux and Open Source by an Accidental Revolutionary. O'Reilly, Sebastopol, CA.

Rogers, Everett M. (1983), Diffusion of Innovation, $3^{\text {rd }}$ ed. New York: The Free Press.

Schiff, A. (2002), The Economics of Open Source Software: A Review of the Early Literature, Review of Network Economics, 1(1), 66-74.

Sethi, S.P. and Thompson, G. L. (2000), Optimal Control Theory: Applications to Management Science and Economics, Second Edition, Kluwer Academic Publishers, Boston. 
Figures

Figure 1: Prices over time in finite and infinite horizon scenarios

Closed Source - Finite $(k=1, w=1)$

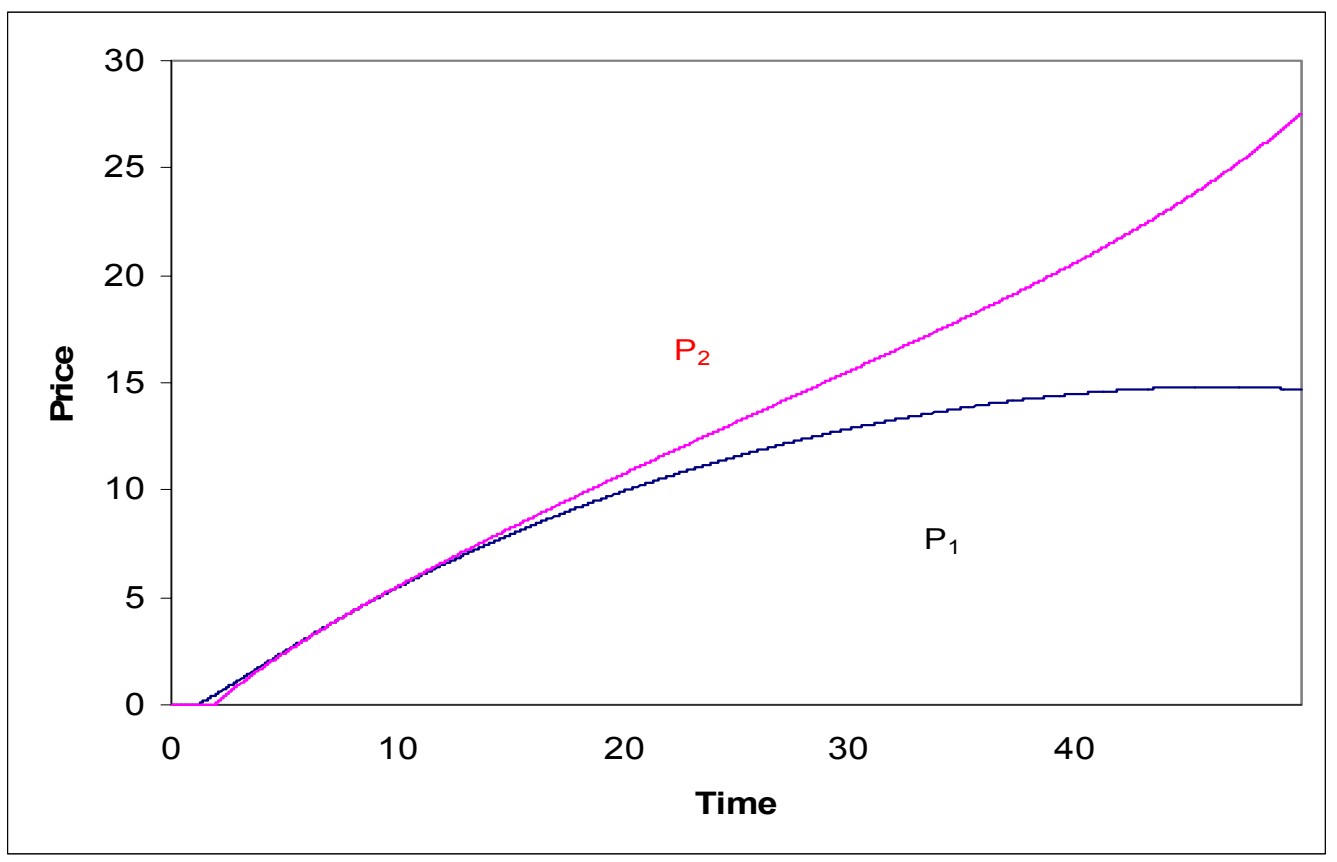

Closed Source - Infinite $(k=1, w=1)$

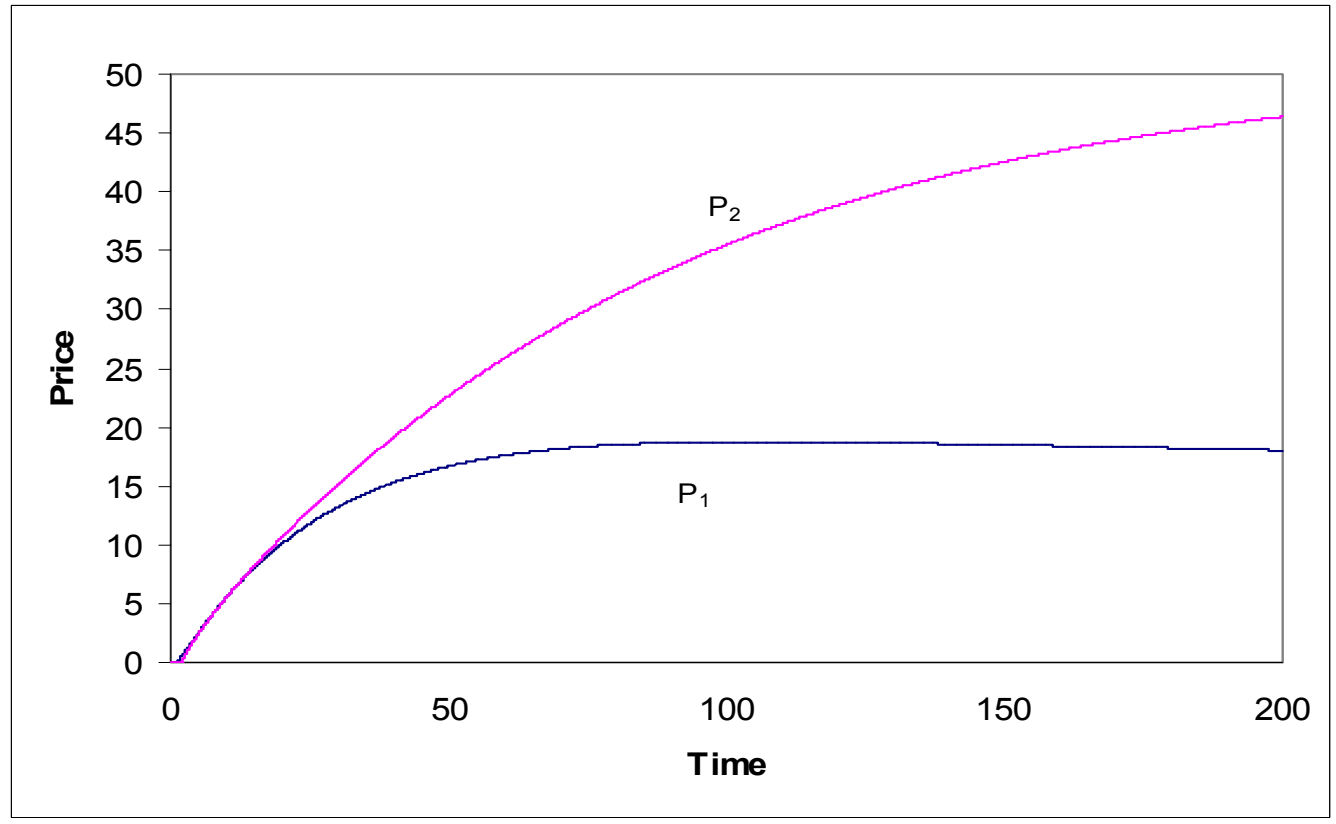




\section{Open Source - Finite $(\alpha=1)$}

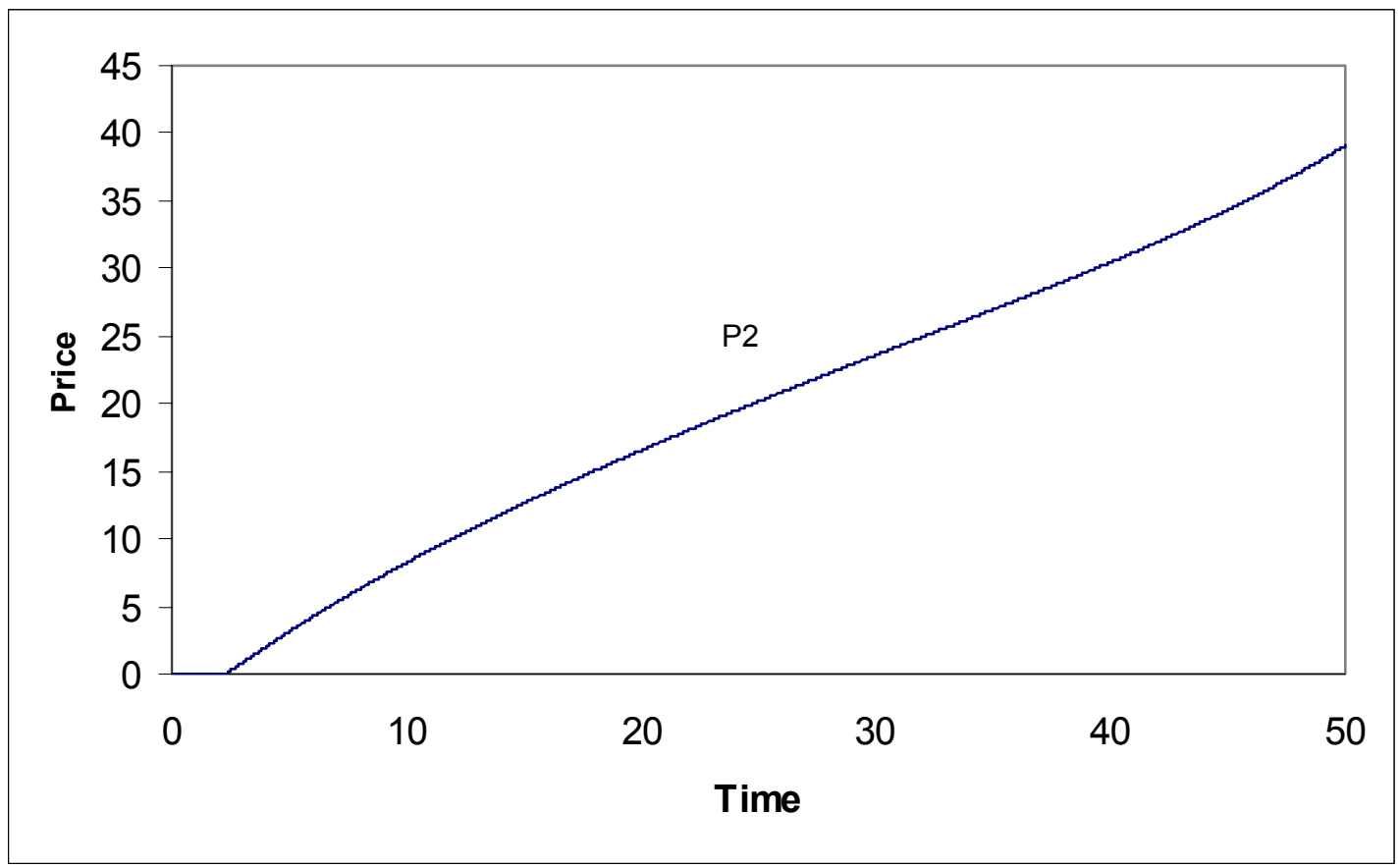

Open Source - Infinite $(\alpha=1)$

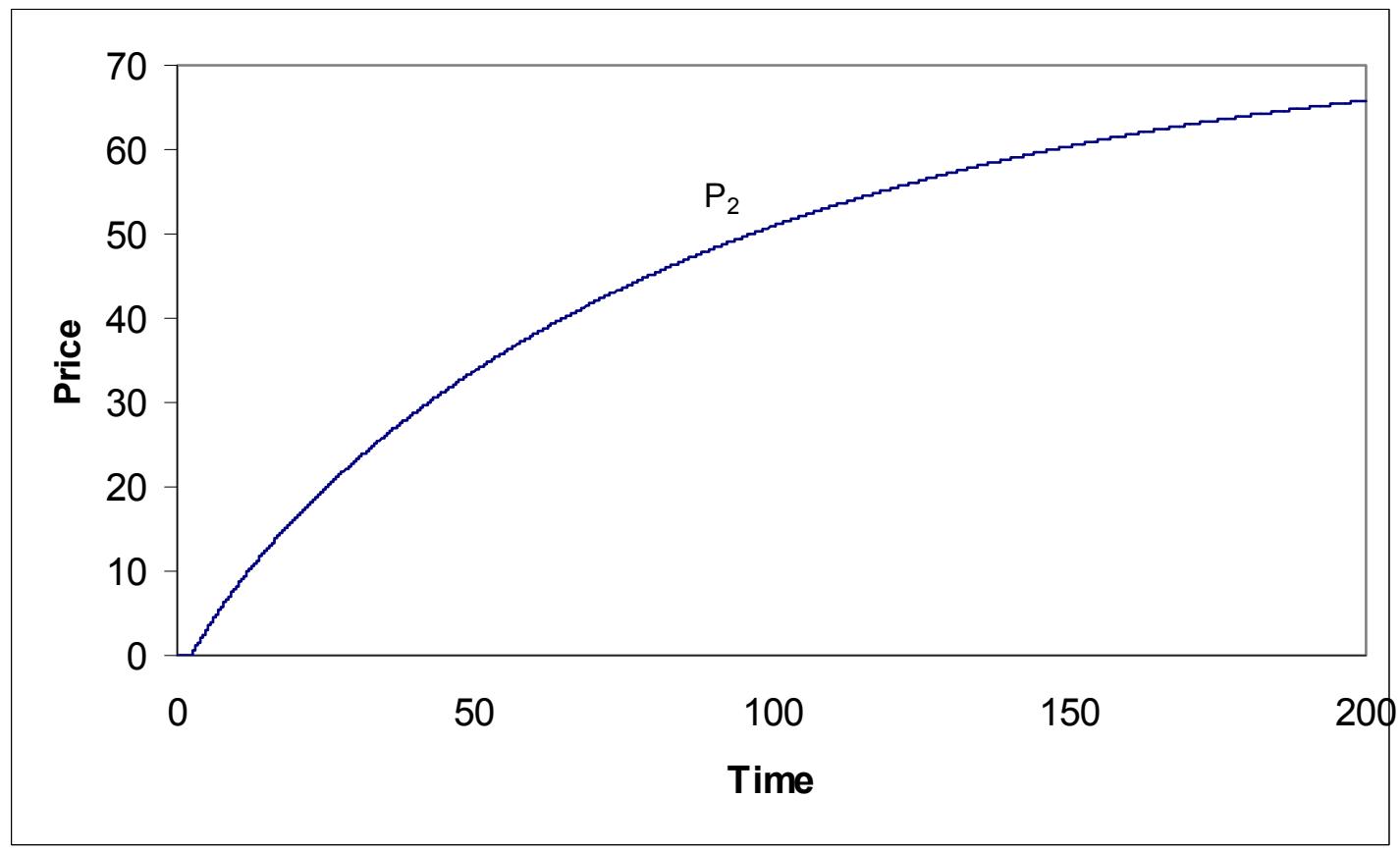


Figure 2: Quality and hiring path for both finite and infinite horizon

\section{Closed Source -- Finite $(k=1, w=1)$}

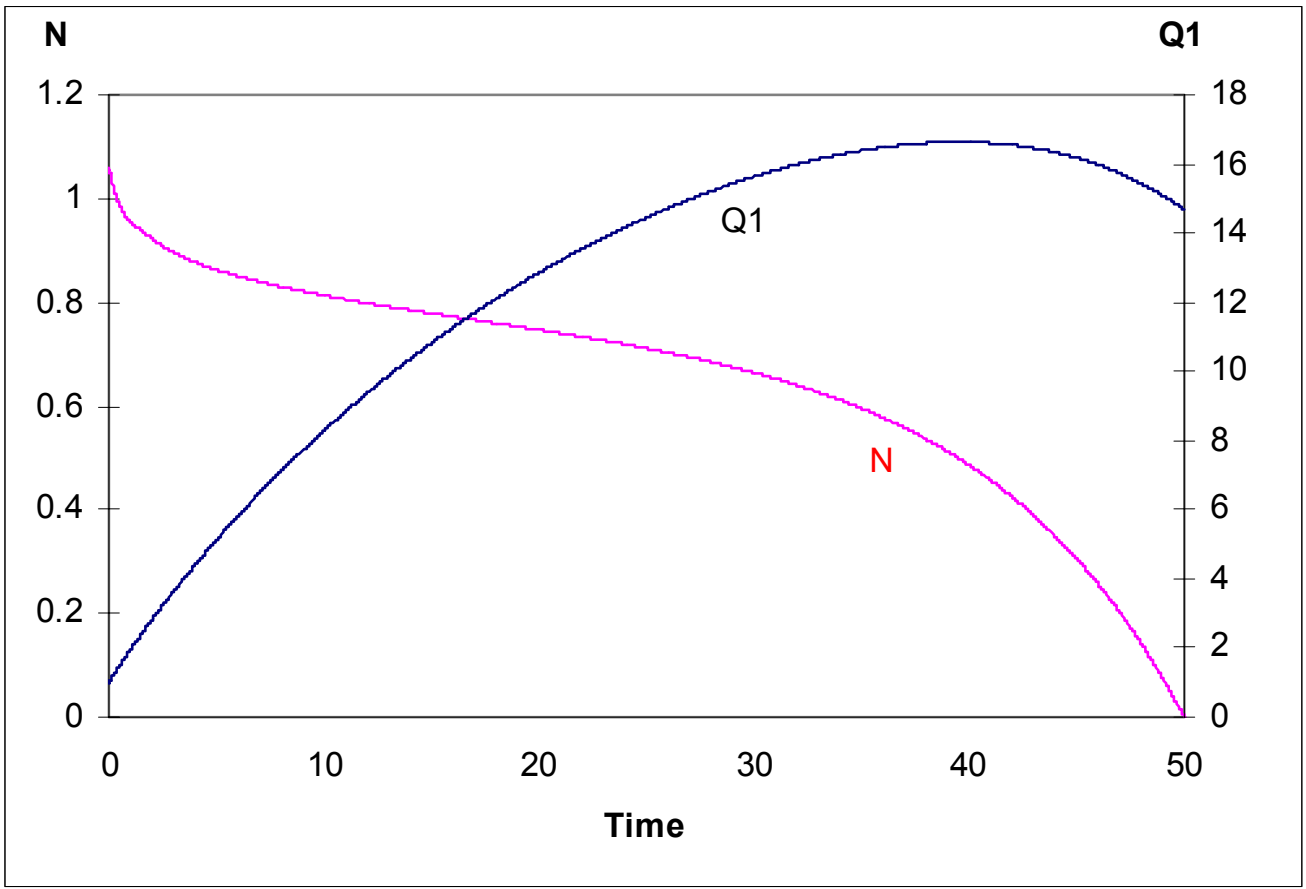

Closed Source - Infinite $(k=1, w=1)$

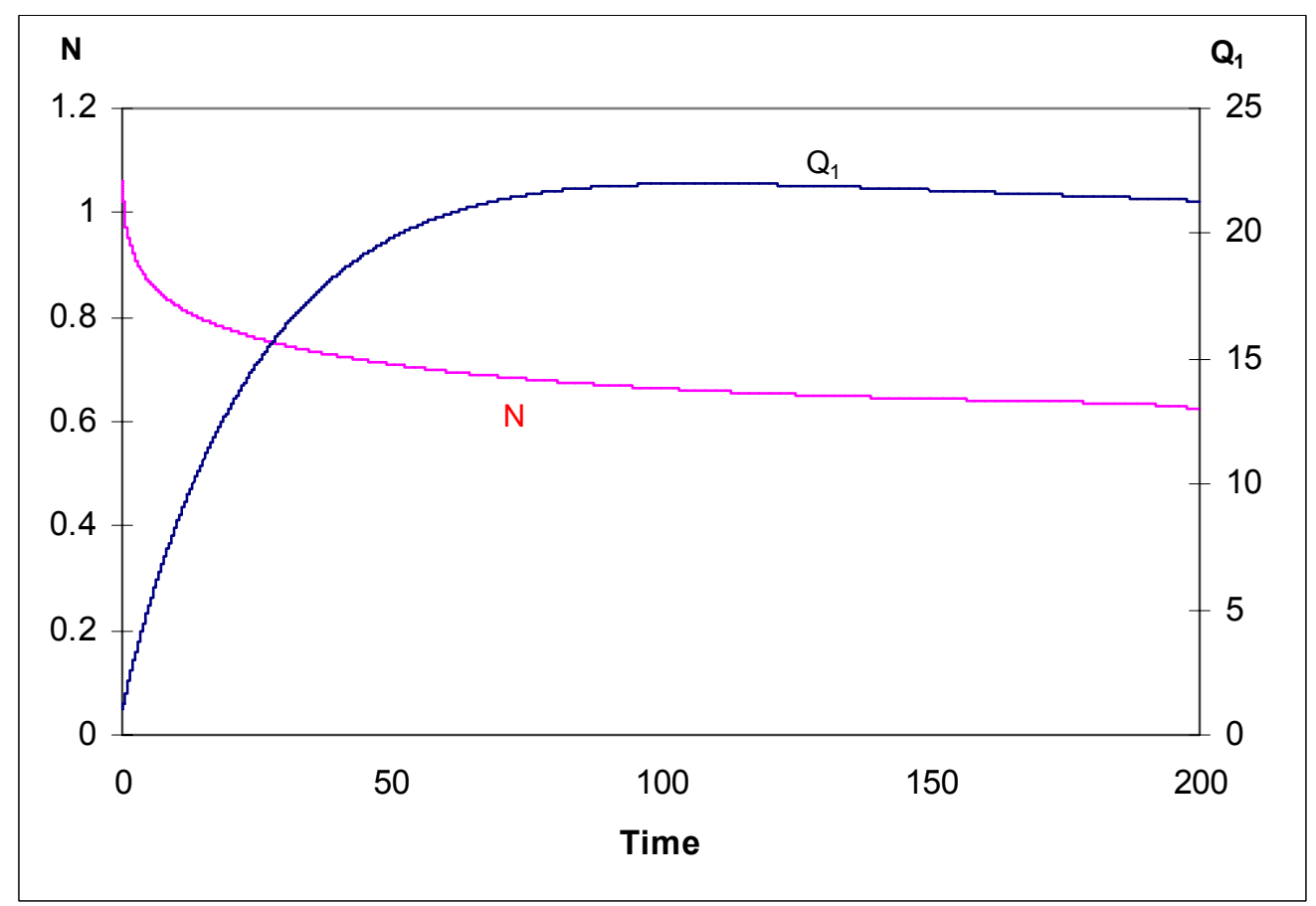



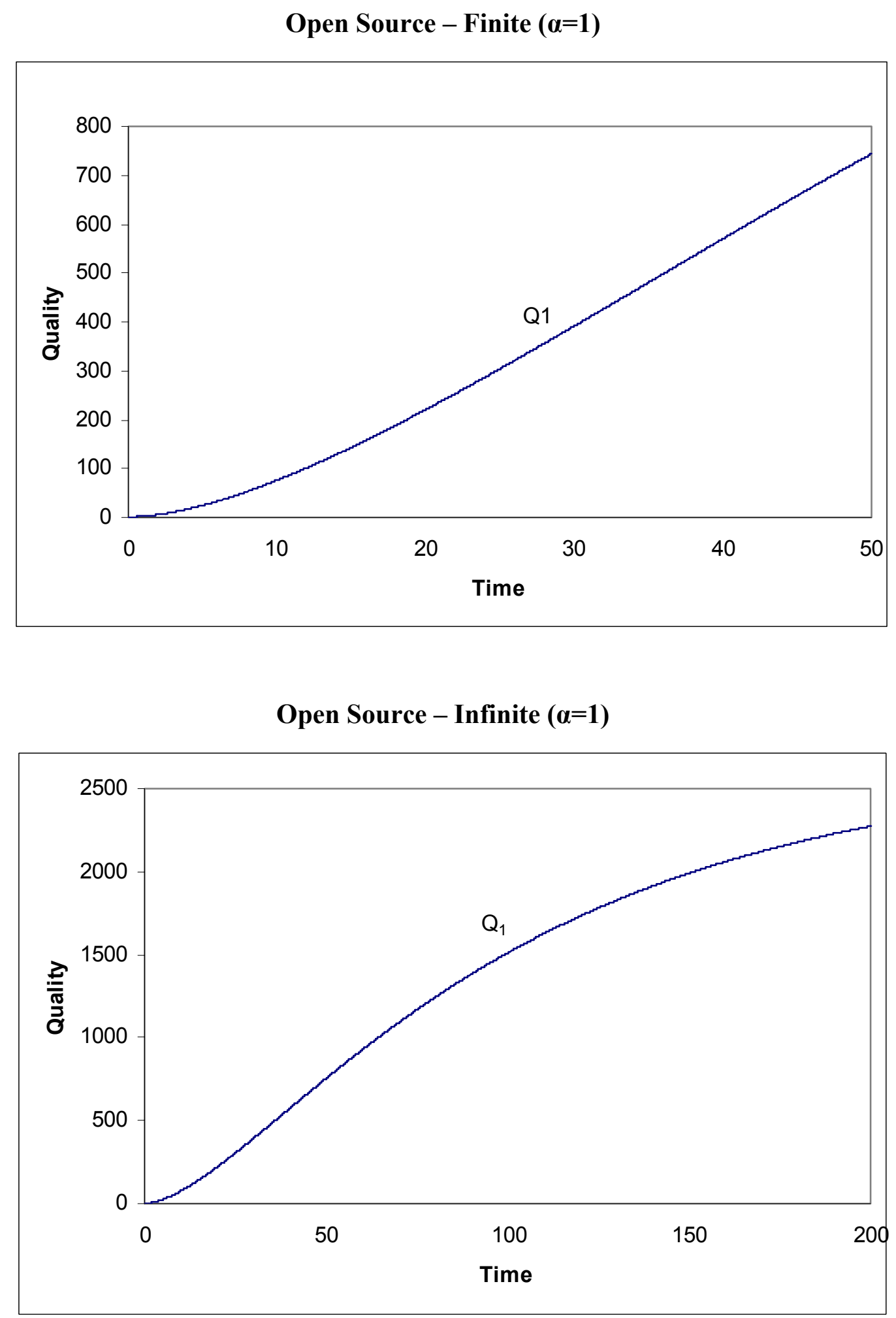
Figure 3: Profit over different values of $\alpha$ for open source and closed source

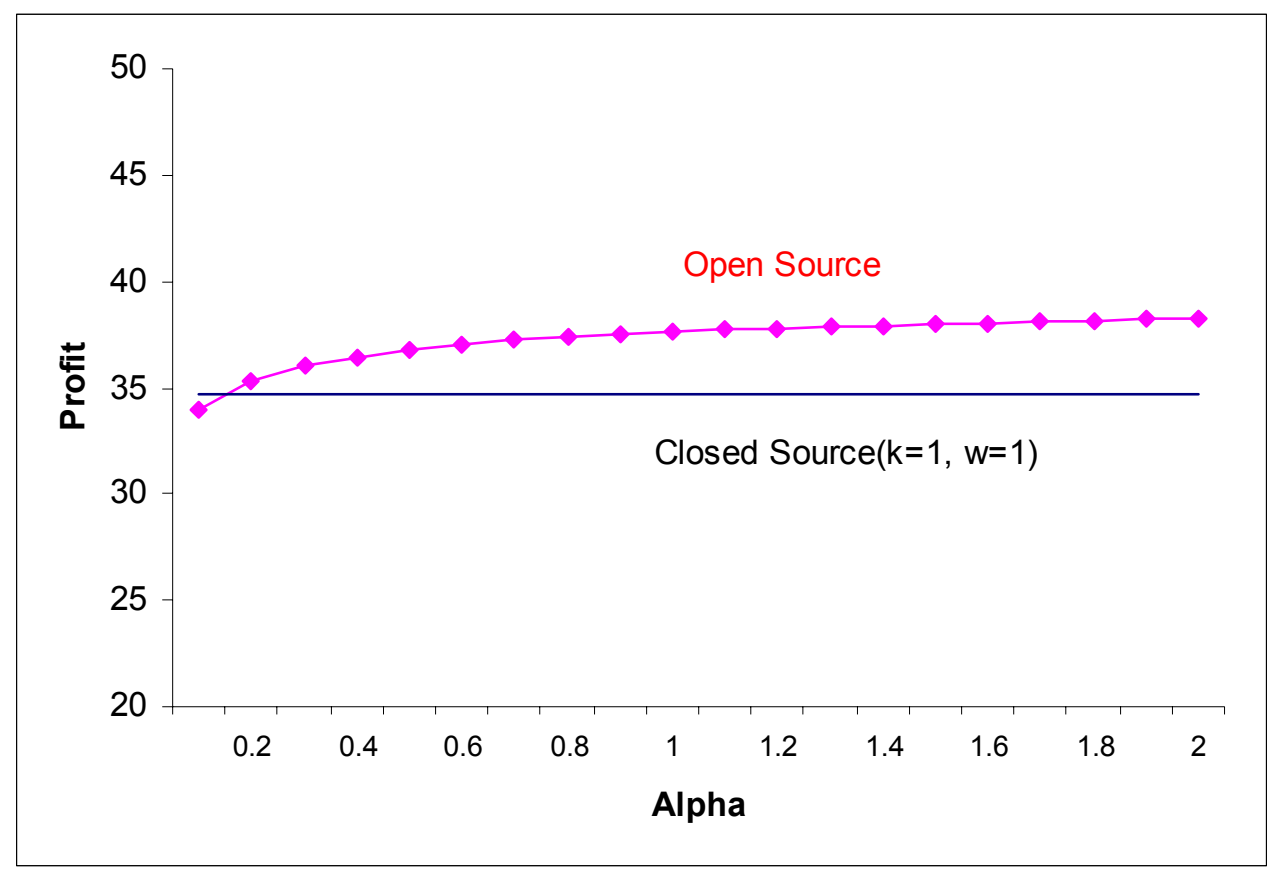

Figure 4: Profit over different values of $k$ for open source and closed source

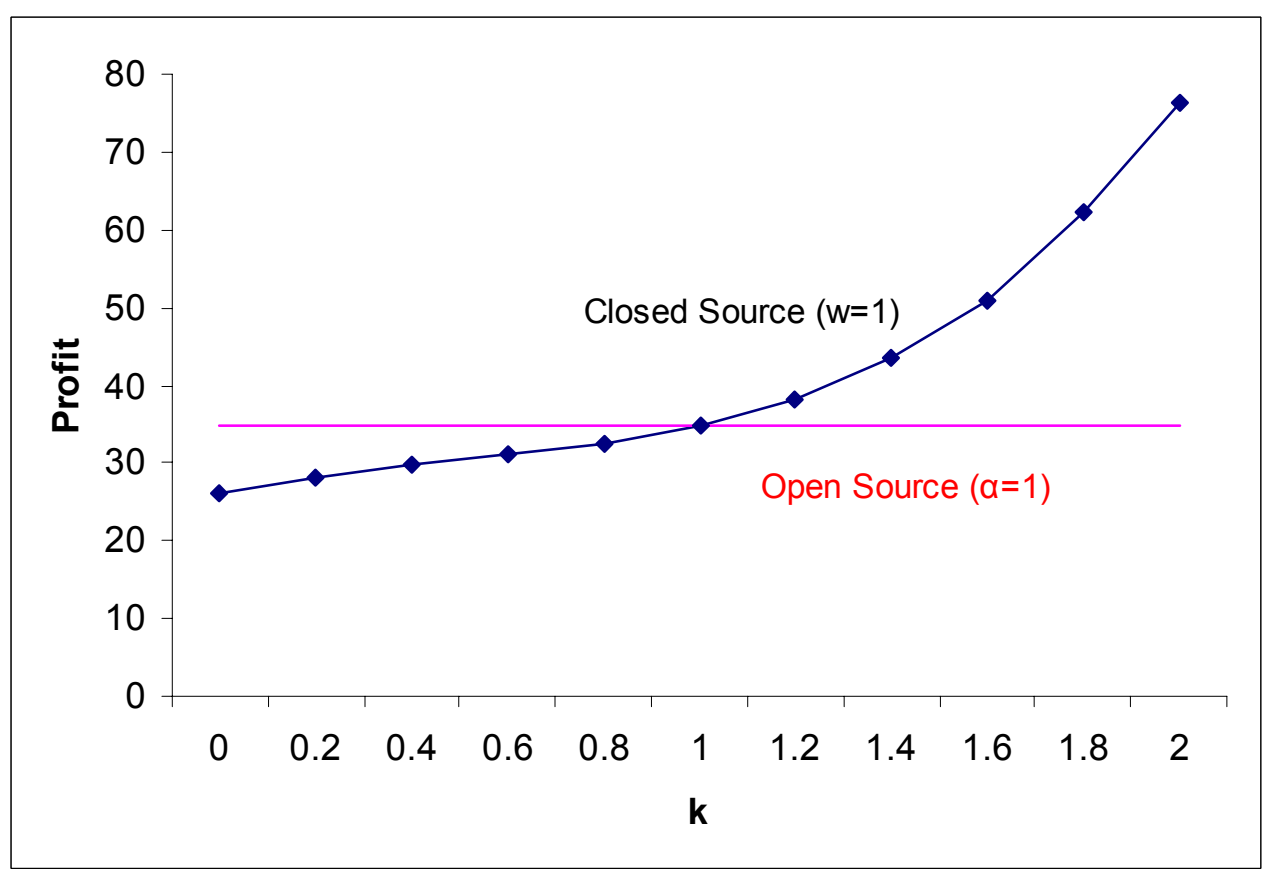


Figure 5: Profit over different values of $w$ for open source and closed source

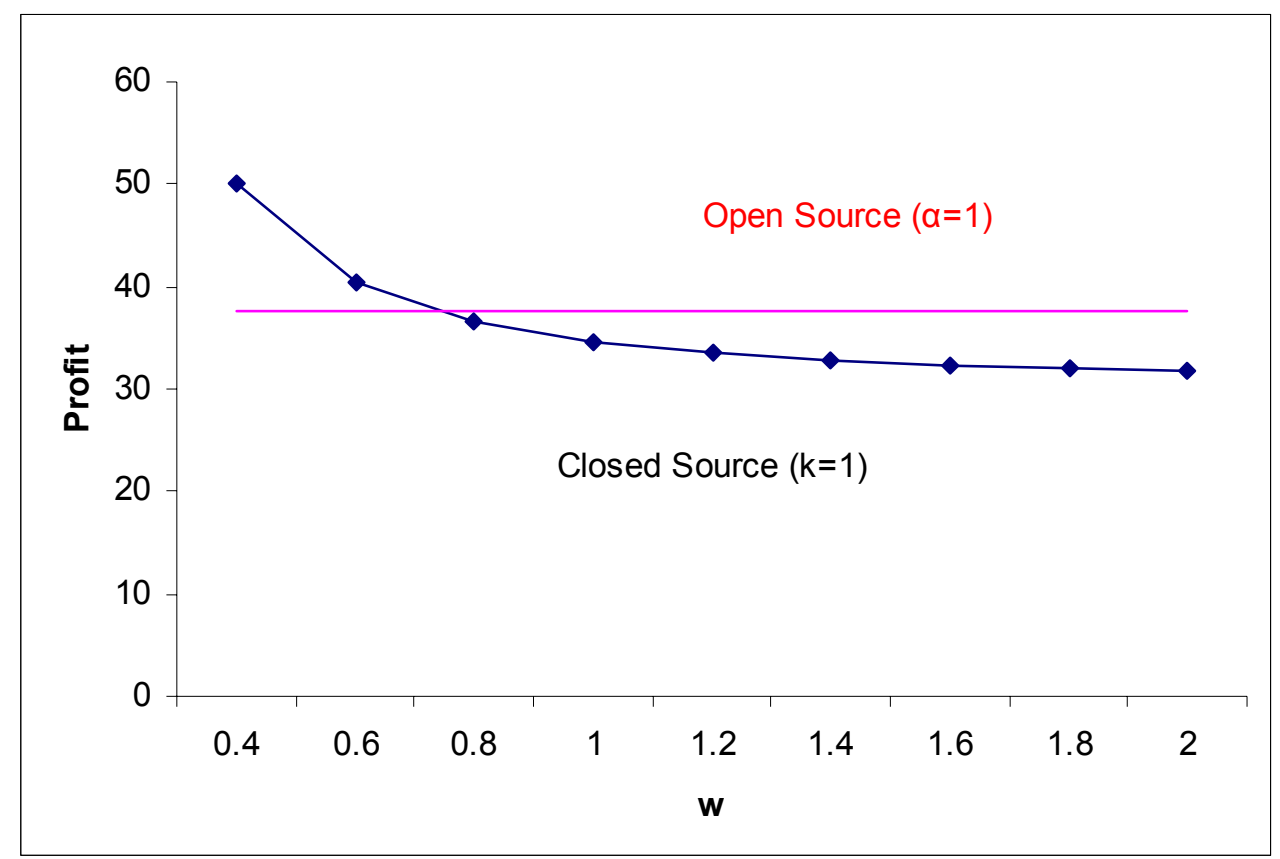

Figure 6. Profit over levels of initial quality $Q_{0}$ (Finite).

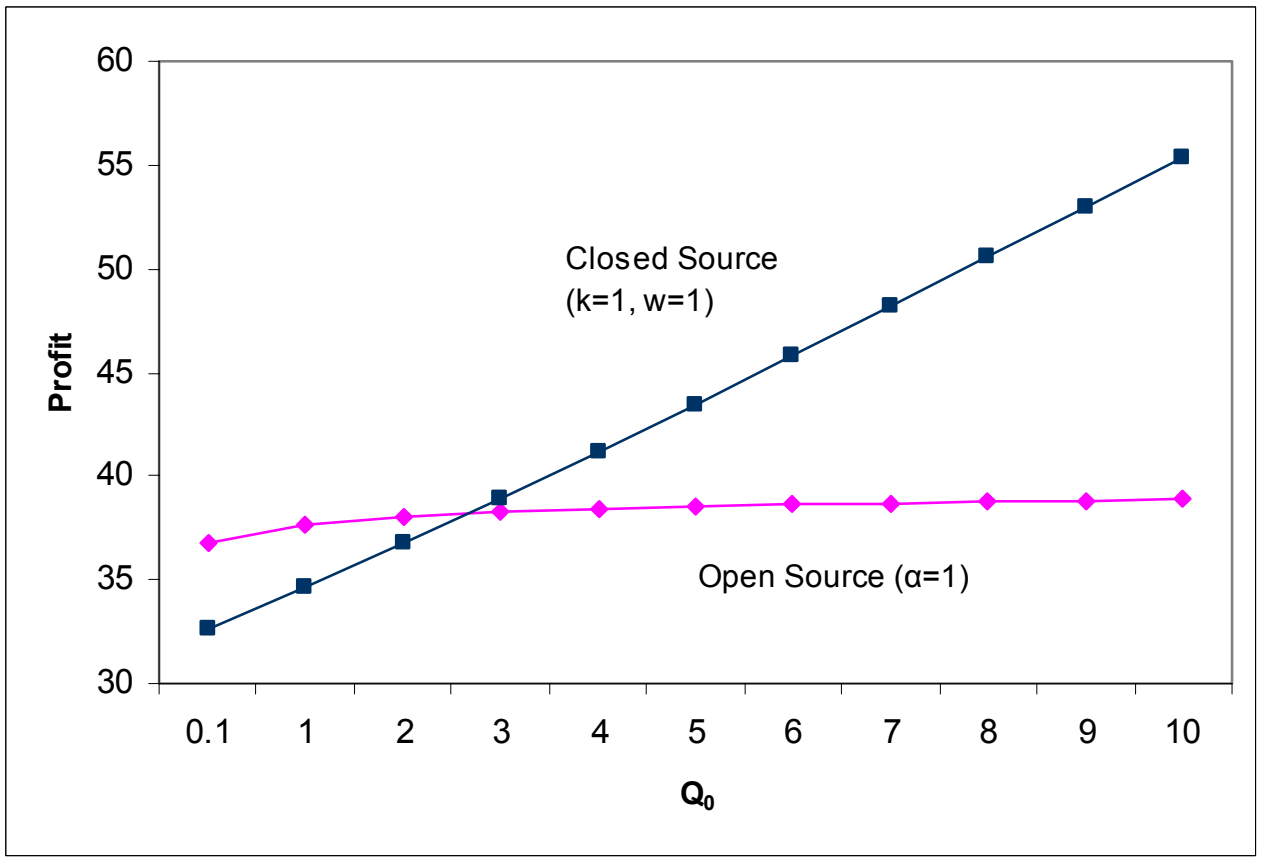


Figure 7: Illustration of Corollary 1.1. Open Source - Infinite Horizon $(\alpha=1)$

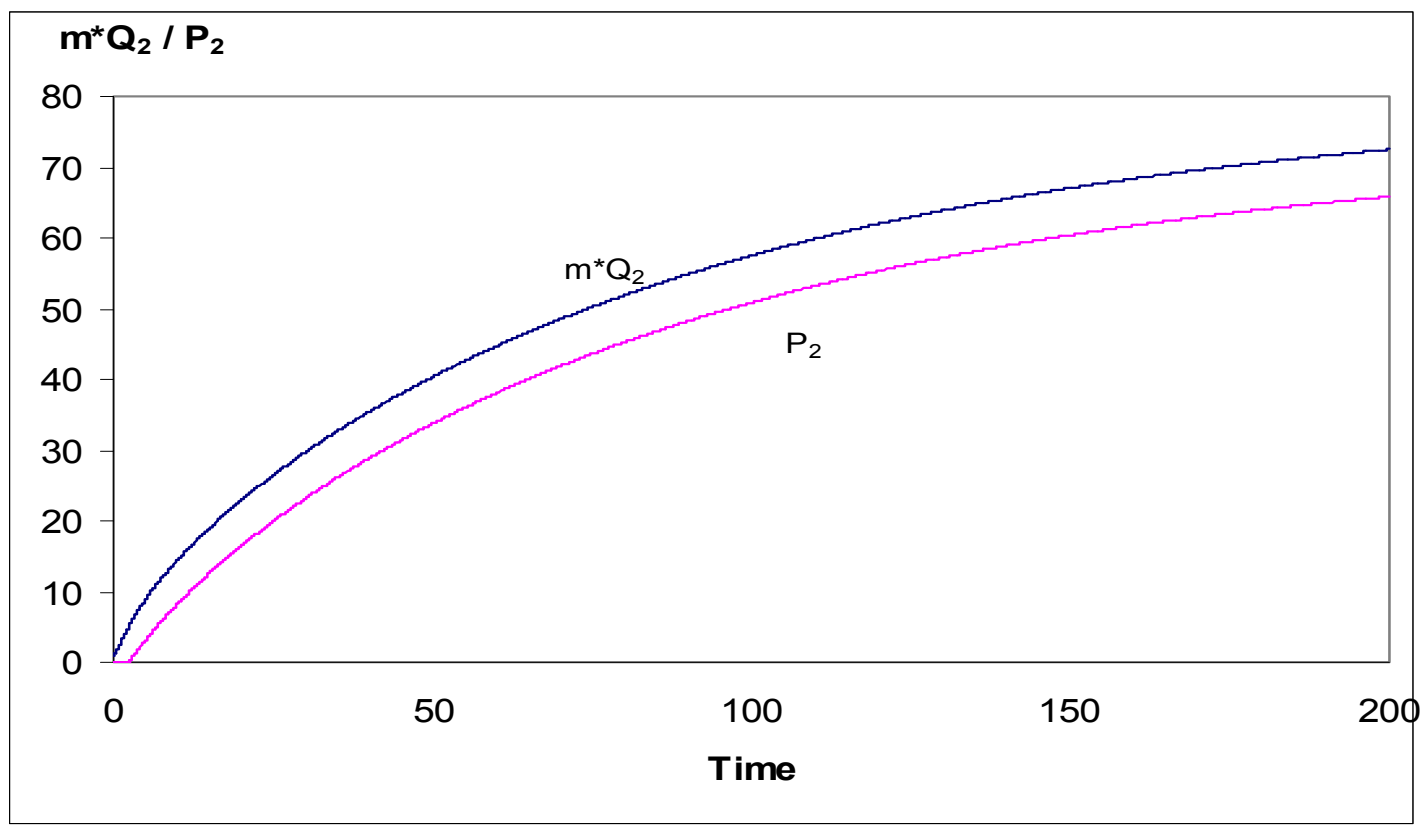

Figure 8: Illustration for Corollary 4.1

$Q_{1}$ versus $P_{1}$ (Closed Source - Infinite) $\quad m * Q_{2}$ versus $P_{2}$ (Closed Source - Infinite) $\mathrm{k}=\mathbf{1}, \mathbf{w}=\mathbf{1}$ $k=1, w=1$
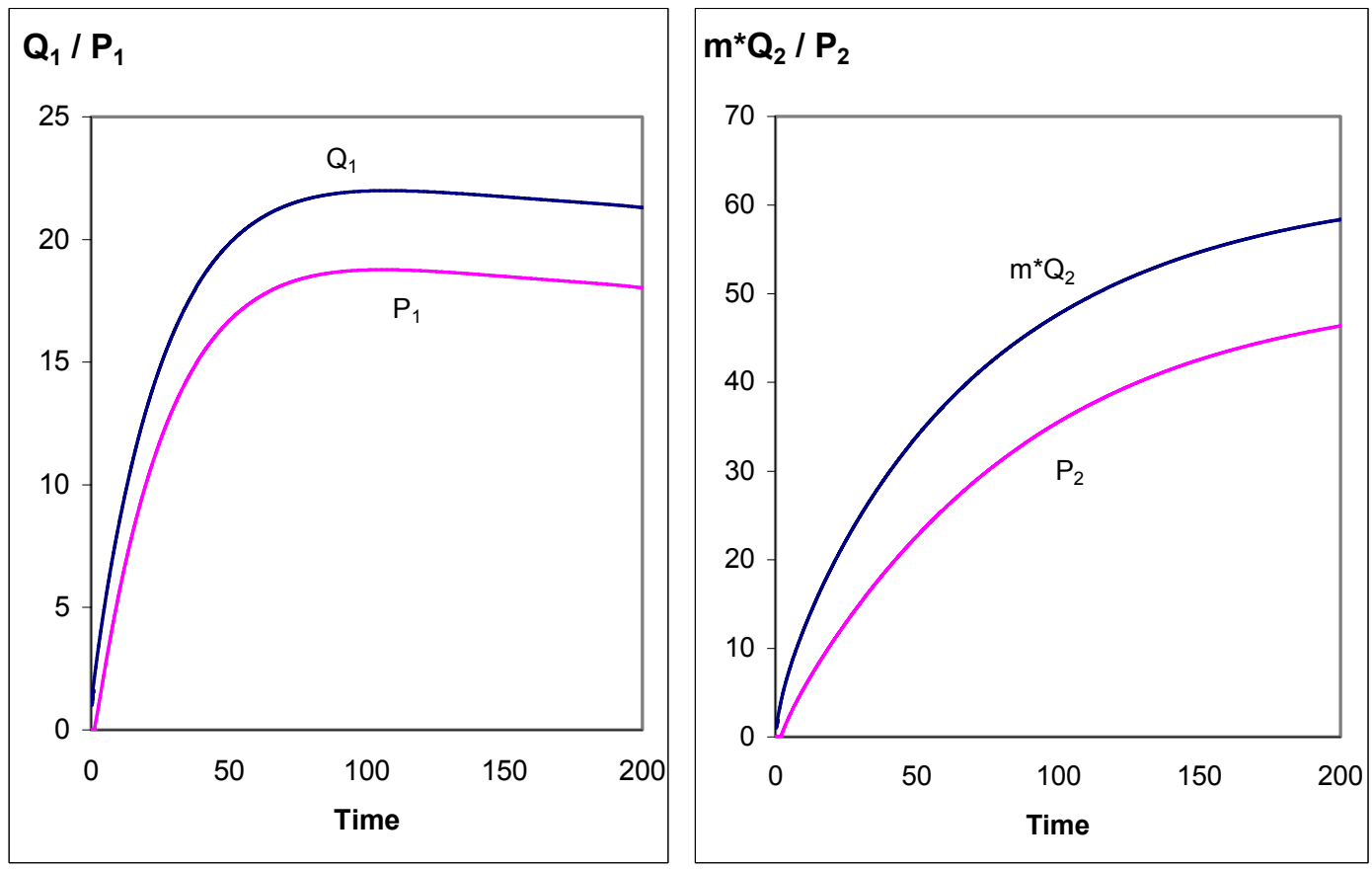
Figure 9: The effects of $Q_{2}$ on $P_{1}$ and $P_{2}$ for closed source (Finite)

$$
Q_{2}=1
$$

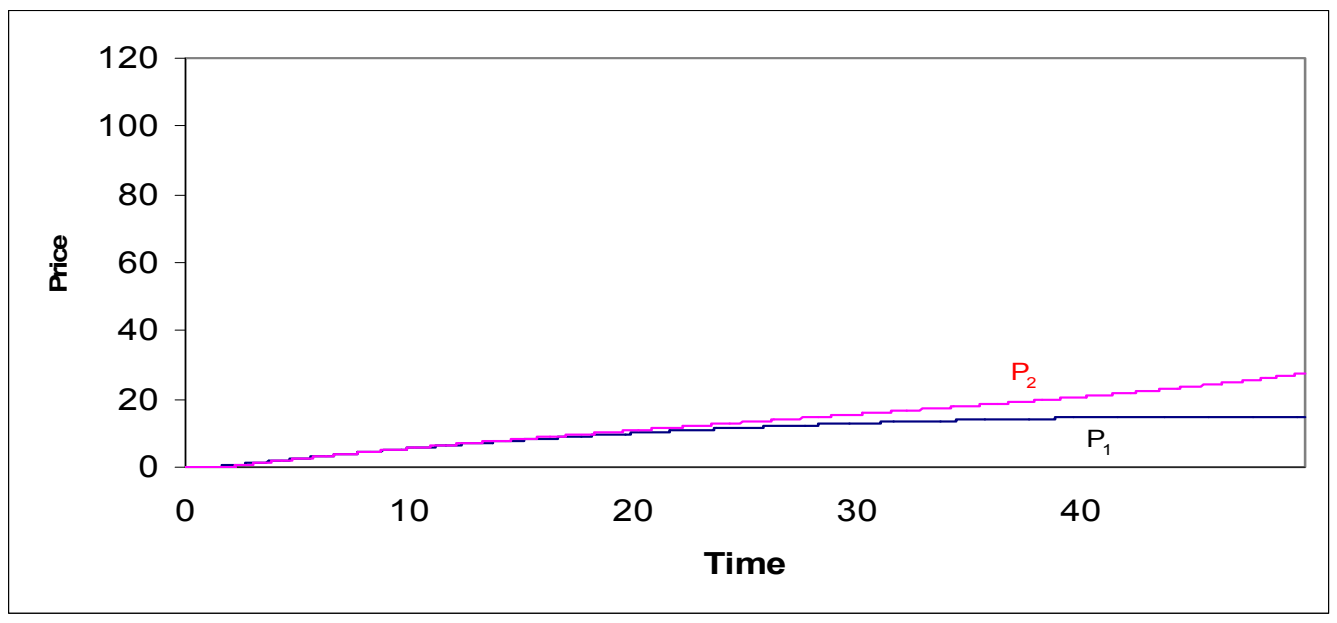

$$
Q_{2}=2
$$

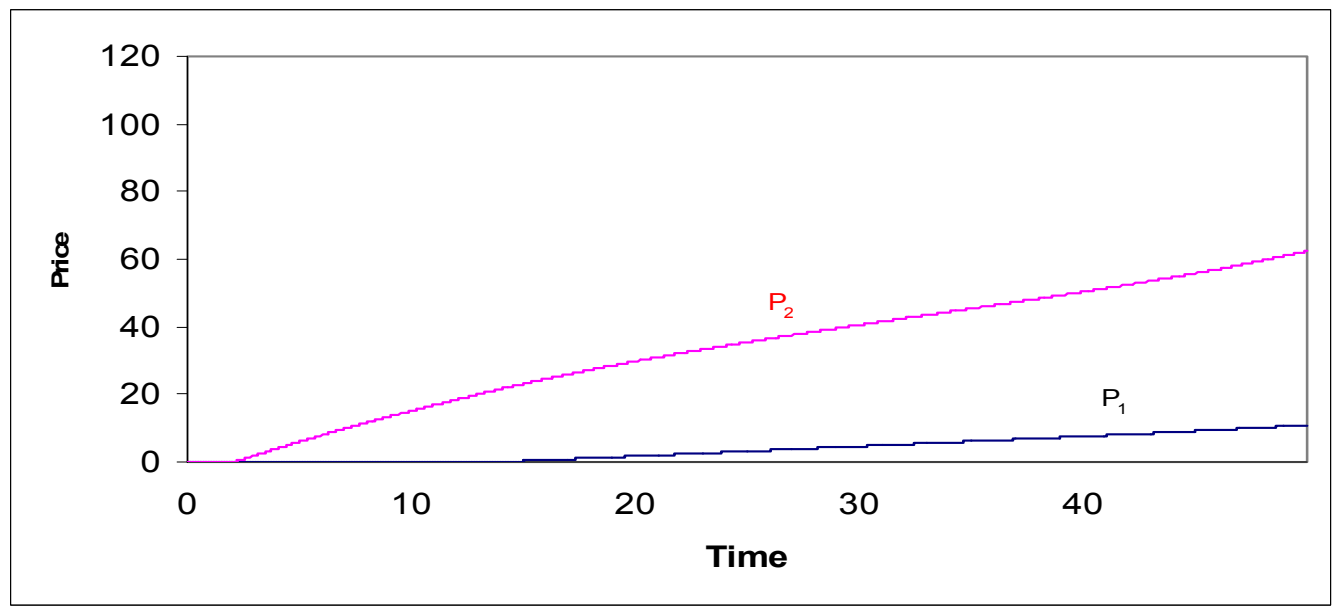

$$
Q_{2}=3
$$

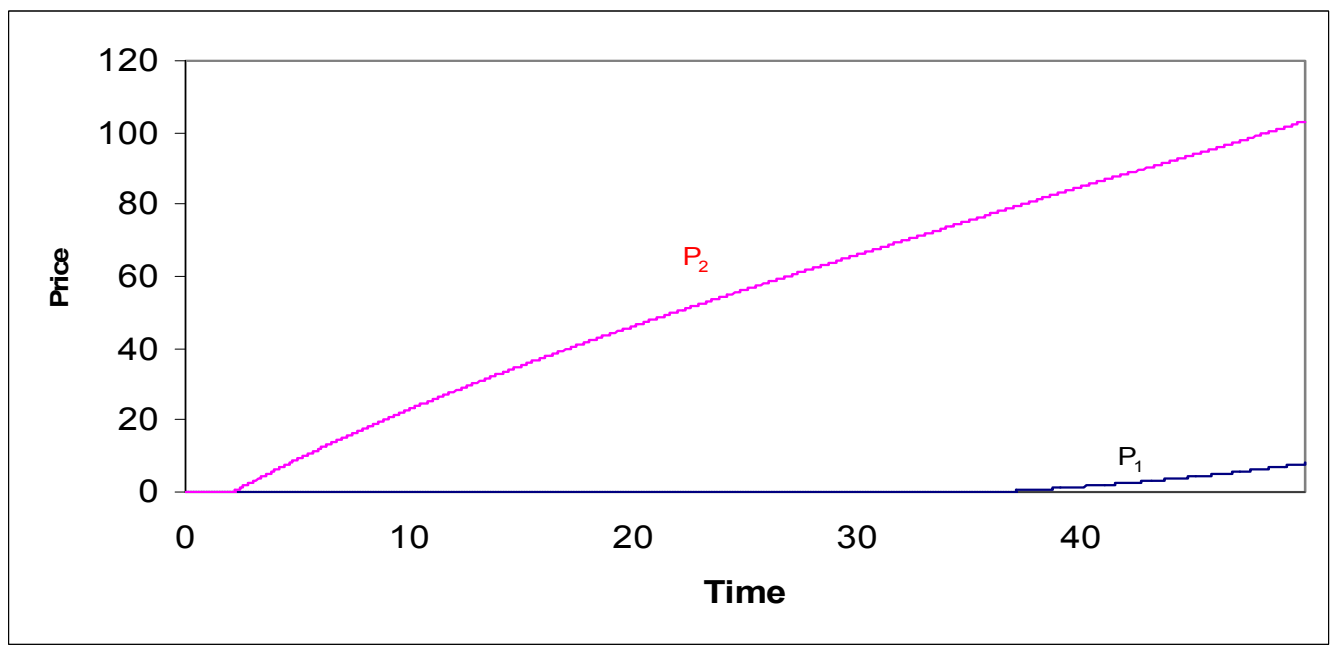




\section{Appendix A: Proof to proposition 1}

(a) Due to network externalities, the price for the complementary product in the open source model starts at zero in order to quickly establish the network's installed base and gradually increases as the network size increases. According to equation (13)

$$
P_{2}=\left\{\begin{array}{cl}
m Q_{2}-\mu\left(a \exp \left(-\frac{c}{Q_{1}}\right)+1\right), & m Q_{2}-\mu\left(a \exp \left(-\frac{c}{Q_{1}}\right)+1\right) \geq 0 \\
0, & m Q_{2}-\mu\left(a \exp \left(-\frac{c}{Q_{1}}\right)+1\right)<0
\end{array}\right.
$$

So at the very beginning, $m Q_{2}-\mu\left(a \exp \left(-\frac{c}{Q_{1}}\right)+1\right)<0$, that is $\mu>\frac{m Q_{2}}{a \exp \left(-\frac{c}{Q_{1}}\right)+1}>0$ $\left(m_{0}>0\right)$. At certain time point, say $\tau, P_{2}(\tau)=m(\tau) Q_{2}-\mu(\tau)\left(a \exp \left(-\frac{c}{Q_{1}(\tau)}\right)+1\right)$.

Take the derivative of $P_{2}$ respect to $m$ at time $\tau$, we have

$$
\left.\frac{\partial P_{2}}{\partial m}\right|_{t=\tau}=Q_{2}-\frac{a c \mu(\tau)}{Q_{1}^{2}(\tau)} \frac{\partial Q_{1}(\tau)}{\partial m(\tau)} e^{-\frac{c}{Q_{1}}}<Q_{2}
$$

also because of continuity $P\left(\tau^{+}\right)<m\left(\tau^{+}\right) Q_{2}$ and $\mu\left(\tau^{+}\right)>0$. Using the same argument, we can prove that $\mu>0$, for all $t \geq 0$. So the marginal benefit from increasing of the size of the network is always positive.

(b) By contradiction.

Suppose at certain time point $\tau, \lambda(\tau)<0$. We have

$$
\dot{\lambda}(\tau)=(\rho+\delta) \lambda(\tau)-\frac{a c \mu}{Q_{1}^{2}} \exp \left(-\frac{c}{Q_{1}}\right) \exp \left(-\frac{P_{2}}{m Q_{2}}\right)<0
$$

then $\lambda<0$, for $t \geq \tau$. So $\lambda(t) \rightarrow-\infty$, as $t \rightarrow \infty$. That means the increasing of the quality would decrease the profit infinitely, which is impossible. So $\lambda>0$, for all $t \geq 0$.

(c) Suppose that the profits for open source and closed source intersect for some value of $\alpha$. Since open source profit is monotonically increasing in $\alpha$ (Use Envelop Theorem and 
equation (8), we have $\left.\frac{d V}{d \alpha}=\frac{\partial L}{\partial \alpha}=\lambda m>0\right)$ and closed source profit is unchanging in $\alpha$, there will be a single point of intersection-a threshold value $\bar{\alpha}$.

\section{Appendix B: Proof to proposition 2}

We try to apply the results by Brock and Scheinkman (1977) to our model to prove the existence of the steady state.

Lemma 1: The profit function in open source problem is concave in $\left(m, P_{2}\right)$.

Proof: Denote $U\left(m, P_{2}\right) \equiv P_{2} D_{2}=P_{2} \exp \left(-\frac{P_{2}}{m Q_{2}}\right)$ as the profit function,

The Hessian of $U\left(m, P_{2}\right)$ is

$$
\left[\begin{array}{ll}
U_{m m} & U_{m P_{2}} \\
U_{P_{2} m} & U_{P_{2} P_{2}}
\end{array}\right]=\left[\begin{array}{cc}
-\frac{P_{2}^{2}}{m^{3} Q_{2}}\left(2-\frac{P_{2}}{m Q_{2}}\right) e^{-\frac{P_{2}}{m Q_{2}}} & \frac{P_{2}}{m^{2} Q_{2}}\left(2-\frac{P_{2}}{m Q_{2}}\right) e^{-\frac{P_{2}}{m Q_{2}}} \\
\frac{P_{2}}{m^{2} Q_{2}}\left(2-\frac{P_{2}}{m Q_{2}}\right) e^{-\frac{P_{2}}{m Q_{2}}} & -\frac{1}{m Q_{2}}\left(2-\frac{P_{2}}{m Q_{2}}\right) e^{-\frac{P_{2}}{m Q_{2}}}
\end{array}\right]
$$

Since $P_{2}<m Q_{2}, U_{m m}<0, U_{P_{2} P_{2}}<0$ and $U_{m m} U_{P_{2} P_{2}}-U_{m P_{2}} U_{P_{2} m}=0$

The concavity follows.

Let

$$
V\left(Q_{0}, m_{0}\right)=\max _{P_{2}} \int_{0}^{\infty} e^{-\rho t} U\left(m, P_{2}\right) d t
$$

Lemma 2: If $U\left(m, P_{2}\right)$ is concave in $\left(m, P_{2}\right)$, then $V(\cdot)$ is concave.

Proof: By the definition of concavity. 
We denote by $\underline{k}=\left(k_{1}, k_{2}\right)=\left(Q_{1}, m\right)$ the vector of the state variables and $\underline{q}=\left(q_{1}, q_{2}\right)=(\lambda, \mu)$ are the vector of the adjoint variables or shadow prices. Brock and Scheinkman (1977, P.190) prove under the assumption of concavity of function $U(k, \dot{k})$ the following

Lemma 3: The shadow price $\underline{q}$ can be written as $\underline{q}(\underline{k})$ and the matrix $\partial \underline{q} / \partial \underline{k}$ is negative semi-definite and symmetric at each $\underline{k}$ where it exists. It exists almost everywhere.

Now we consider the system of ordinary differential equations in the plane:

$$
\begin{aligned}
& \dot{k_{1}}=H_{q_{q}}\left(q_{1}(\underline{k}), q_{2}(\underline{k}), k_{1}, k_{2}\right)=H_{q_{1}}(\underline{q}, \underline{k}) \equiv F_{21}(\underline{k}) \\
& \dot{k_{1}}=H_{q_{2}}\left(q_{1}(\underline{k}), q_{2}(\underline{k}), k_{1}, k_{2}\right)=H_{q_{2}}(\underline{q}, \underline{k}) \equiv F_{22}(\underline{k}) \\
& \underline{k}(0)=\underline{k}_{0}
\end{aligned}
$$

The Jacobian matrix of $F_{2}$ is

$$
J_{F_{2}}=H_{q q} \frac{\partial^{2} V}{\partial \underline{k}^{2}}+H_{q k}
$$

where $\frac{\partial \underline{q}}{\partial \underline{k}}=\frac{\partial^{2} V}{\partial \underline{k}^{2}}$ and $H_{q}=\frac{\partial H}{\partial \underline{q}} ; H_{k}=\frac{\partial H}{\partial \underline{k}}$.

Hsu and Meyer (1968, p.164) proved the following theorem:

Lemma 4: If the trace $J_{F_{2}}(\underline{k})$ does not change sign for all $\underline{k}$, then there are no limit cycle for $(*)$.

It can now be proven using the result of Brock and Scheinkman (1977, P.190-193):

Lemma 5: Let $A, B$ be two $2 \times 2$ real matrices that are symmetric; $A$ positive definite and $B$ negative definite, then

(i) $\operatorname{trace}(A B)<0$.

If only semi-positive definiteness and semi-negative definiteness holds, then

(ii) $\operatorname{trace}(A B) \leq 0$ 
If neither $A$ nor lis 0 and trace $(A B)=0$, then

(iii) $A$ and $B$ are both singular.

Theorem 1: Assume that there is a bound $M\left(\underline{k}_{0}\right)$ such that

$$
\left\|\underline{k}\left(t / \underline{k}_{0}\right)\right\| \leq M\left(\underline{k}_{0}\right)
$$

For all $t \geq 0$. Also assume $V^{\prime \prime}(\underline{k}) \neq 0, H_{11}$ positive definite, and trace $H_{12}(\underline{k}) \leq 0$, then $\underline{k}\left(t / \underline{k}_{0}\right) \rightarrow \underline{\bar{k}}, t \rightarrow \infty$, when $\underline{\underline{k}}$ is a rest point of $(*)$.

Now we apply these results to our open source model under the assumption $P_{2}>0$.

We have

$$
\begin{aligned}
& \dot{k}_{1} \equiv \dot{Q}_{1}=\frac{\partial H}{\partial \lambda}=\alpha m-\delta Q_{1} \equiv H_{q_{1}} \\
& \dot{k}_{2} \equiv \dot{m}=a \exp \left(-\frac{c}{Q_{1}}\right) \exp \left(-\frac{P_{2}(\underline{k}, \underline{q})}{m Q_{2}}\right)+\exp \left(-\frac{P_{2}(\underline{k}, \underline{q})}{m Q_{2}}\right)-\varepsilon m \equiv H_{q_{2}}
\end{aligned}
$$

So

$$
\begin{aligned}
H_{q q} & =\left[\begin{array}{ll}
\frac{\partial H_{q_{1}}}{\partial \lambda} & \frac{\partial H_{q_{1}}}{\partial \mu} \\
\frac{\partial H_{q_{2}}}{\partial \lambda} & \frac{\partial H_{q_{2}}}{\partial \mu}
\end{array}\right]=\left[\begin{array}{ccc}
0 & 0 \\
m Q_{2} & \left(a e^{-\frac{c}{Q_{1}}}+1\right) e^{-\frac{P_{2}}{m Q_{2}}} \frac{\partial P_{2}}{\partial \lambda} & -\frac{1}{m Q_{2}}\left(a e^{-\frac{c}{Q_{1}}}+1\right) e^{-\frac{P_{2}}{m Q_{2}}} \frac{\partial P_{2}}{\partial \mu}
\end{array}\right] \\
H_{q k} & =\left[\begin{array}{ll}
\frac{\partial H_{q_{1}}}{\partial Q_{1}} & \frac{\partial H_{q_{1}}}{\partial m} \\
\frac{\partial H_{q_{2}}}{\partial Q_{1}} & \frac{\partial H_{q_{2}}}{\partial m}
\end{array}\right] \\
& =\left[\begin{array}{lll}
\frac{a c}{Q_{1}^{2}} e^{-\frac{c}{Q_{1}}-\frac{P_{2}}{m Q_{2}}}-\frac{1}{m Q_{2}}\left(a e^{-\frac{c}{Q_{1}}}+1\right) e^{-\frac{P_{2}}{m Q_{2}}} \frac{\partial P_{2}}{\partial Q_{1}} & \left(\frac{P_{2}}{m^{2} Q_{2}}-\frac{1}{m Q_{2}} \frac{\partial P_{2}}{\partial m}\right)\left(a e^{-\frac{c}{Q_{1}}}+1\right) e^{-\frac{P_{2}}{m Q_{2}}}-\varepsilon
\end{array}\right]
\end{aligned}
$$

We need to derive $\frac{\partial P_{2}}{\partial \lambda}, \frac{\partial P_{2}}{\partial \mu}, \frac{\partial P_{2}}{\partial m}$.

We have 


$$
P_{2}=m Q_{2}-\mu\left(a \exp \left(-\frac{c}{Q_{1}}\right)+1\right)
$$

So $\quad \frac{\partial P_{2}}{\partial \lambda}=0, \frac{\partial P_{2}}{\partial \mu}=-\left(a \exp \left(-\frac{c}{Q_{1}}\right)+1\right)<0$ and $\frac{\partial P_{2}}{\partial m}=Q_{2}$

We have

$$
H_{q q}=\left[\begin{array}{ll}
0 & 0 \\
0 & \frac{1}{m Q_{2}}\left(a e^{-\frac{c}{Q_{1}}}+1\right)^{2} e^{-\frac{P_{2}}{m Q_{2}}}>0
\end{array}\right] \text {, so } H_{q q} \text { is symmetric and positive semi- }
$$

definite. In addition $V$ is concave so $\frac{\partial^{2} V}{\partial \underline{k}^{2}}$ negative semi-definiteness and symmetry follows.

$$
J_{F_{2}}=H_{q q} \frac{\partial^{2} V}{\partial \underline{k}^{2}}+H_{q k}
$$

By lemma 5,

$$
\operatorname{trace}(\mathrm{J})=\operatorname{trace}\left(H_{q q} \frac{\partial^{2} V}{\partial \underline{k}^{2}}\right)+\operatorname{trace}\left(H_{q k}\right) \leq \operatorname{trace}\left(H_{q k}\right)
$$

Compute trace $\left(H_{q k}\right)=-\delta+\left(\frac{P_{2}}{m^{2} Q_{2}}-\frac{1}{m Q_{2}} \frac{\partial P_{2}}{\partial m}\right)\left(a e^{-\frac{c}{Q_{1}}}+1\right) e^{-\frac{P_{2}}{m Q_{2}}}-\varepsilon$

$$
=-\delta-\frac{1}{m^{2} Q_{2}}\left(m Q_{2}-P_{2}\right)\left(a e^{-\frac{c}{Q 1}}+1\right) e^{-\frac{P 2}{m Q 2}}-\varepsilon<0
$$

Theorem 2: In open source case, if

(a) at the steady state, $\operatorname{trace}\left(H_{q k}\right)<0$

and (b) there is a closed curve $\Gamma$ containing the steady state such that

$$
\underline{\dot{k}}=H_{q}(q(\underline{k}), \underline{k})
$$

'point inward' on $\Gamma$, then, the steady state is locally asymptotically stable (except for hairline cases). 
The condition b) is saying that $\dot{k}_{i}>0$ if $k_{i}$ is small enough and $\dot{k}_{i}<0$ if $k_{i}$ is large enough. In our case, $\dot{Q}_{1}>0$ if $Q_{1}$ is small enough and $\dot{Q}_{1}<0$ if $Q_{1}$ is large enough; and $\dot{m}>0$ if $m$ is small enough and $\dot{m}<0$ if $m$ is large enough. This can be easily shown.

\section{Appendix C: Proof to proposition 3}

We reduce equation (15) - (19) to one equation and show that it has a unique solution. From (17),

$$
\lambda=\frac{a c \mu}{(\rho+\delta) Q_{1}^{2}} \exp \left(-\frac{c}{Q_{1}}-\frac{P_{2}}{m Q_{2}}\right)
$$

From (19),

$$
\frac{P_{2}}{m Q_{2}}=1-\frac{\mu}{m Q_{2}}\left(a \exp \left(-\frac{c}{Q_{1}}\right)+1\right)
$$

From (16),

$$
\frac{P_{2}}{m Q_{2}}=\ln \frac{a \exp \left(-\frac{c}{Q_{1}}\right)+1}{\varepsilon m}
$$

Let $(\mathrm{C} 2)=(\mathrm{C} 3)$,

$$
\mu=\frac{m Q_{2}\left[1-\ln \frac{a \exp \left(-\frac{c}{Q_{1}}\right)+1}{\varepsilon m}\right]}{a \exp \left(-\frac{c}{Q_{1}}\right)+1}
$$

From (15),

$$
m=\frac{\delta Q_{1}}{\alpha}
$$

Put (19), (C1), (C4) and (C5) into (18), we can get

$$
f(Q) \equiv\left[\rho+\varepsilon-\frac{a c \varepsilon \delta}{(\rho+\delta)\left(a+e^{\frac{c}{Q_{1}}}\right) Q_{1}}\right]-\left[\rho+2 \varepsilon-\frac{a c \varepsilon \delta}{(\rho+\delta)\left(a+e^{\frac{c}{Q_{1}}}\right) Q_{1}}\right] \ln \frac{\alpha\left(a e^{-\frac{c}{Q_{1}}}+1\right)}{\varepsilon \delta Q_{1}}=0
$$




$$
\begin{aligned}
& \text { When } Q_{1} \rightarrow 0, \lim _{Q_{1} \rightarrow 0} f\left(Q_{1}\right)<0 \\
& \qquad \begin{array}{l}
Q_{1} \rightarrow \infty, \lim _{Q_{1} \rightarrow \infty} f\left(Q_{1}\right)>0 \\
\frac{\partial f\left(Q_{1}\right)}{\partial Q_{1}}>0 \quad \text { For } \forall Q_{1}>0
\end{array}
\end{aligned}
$$

So there exists a unique $\overline{Q_{1}}$. Therefore, there exist unique $\bar{m}, \bar{P}_{2}, \bar{\lambda}, \bar{\mu}$.

\section{Appendix D: Proof to proposition 4}

(a) By contradiction.

Suppose at certain time point $\tau, \mu(\tau)<0$. From equation (29),

$$
P_{2}=\left\{\begin{array}{cl}
m Q_{2}-\mu-\left(P_{1}+a \mu\right) \exp \left(-\frac{P_{1}+c}{Q_{1}}\right), & m Q_{2}-\mu-\left(P_{1}+a \mu\right) \exp \left(-\frac{P_{1}+c}{Q_{1}}\right) \geq 0 \\
0, & m Q_{2}-\mu-\left(P_{1}+a \mu\right) \exp \left(-\frac{P_{1}+c}{Q_{1}}\right)<0
\end{array}\right.
$$

(i) If $m(\tau) Q_{2}-\mu(\tau)-\left(P_{1}(\tau)+a \mu(\tau)\right) \exp \left(-\frac{P_{1}(\tau)+c}{Q_{1}(\tau)}\right) \geq 0$,

then $P_{2}(\tau)=m(\tau) Q_{2}-\mu(\tau)-\left(P_{1}(\tau)+a \mu(\tau)\right) \exp \left(-\frac{P_{1}(\tau)+c}{Q_{1}(\tau)}\right) \geq 0$. Put $P_{2}(\tau)$ into (23) we have

$$
\dot{\mu}(\tau)=(\rho+\varepsilon) \mu(\tau)-\frac{P_{2}(\tau)}{m(\tau)} \exp \left(-\frac{P_{2}(\tau)}{m(\tau) Q_{2}}\right)<0
$$

We have $\mu(t)<0$, for $t \geq \tau$. So $\mu(t) \rightarrow-\infty$, as $t \rightarrow \infty$. That means the increasing of the size of the network of users would decrease the profit infinitely, which is impossible.

(ii) If $m(\tau) Q_{2}-\mu(\tau)-\left(P_{1}(\tau)+a \mu(\tau)\right) \exp \left(-\frac{P_{1}(\tau)+c}{Q_{1}(\tau)}\right)<0$, then $P_{2}(\tau)=0$. Put $P_{2}(\tau)$ into (23), we have

$$
\dot{\mu}(\tau)=(\rho+\varepsilon) \mu(\tau)<0
$$


Same as the previous case, $\mu(t)<0$, for $t \geq \tau$. So $\mu(t) \rightarrow-\infty$, as $t \rightarrow \infty$. That means the increasing of the size of the network of users would decrease the profit infinitely, which is impossible. So $\mu(t)>0$, for all $t \geq 0$. This means that the marginal benefit from increasing of the size of the network is always positive.

(b) By contradiction.

Suppose at certain time point $\tau, \lambda(\tau)<0$. We have

$$
\dot{\lambda}(\tau)=(\rho+\delta) \lambda(\tau)-\frac{\left(P_{1}+a \mu\right)\left(P_{1}+c\right)}{Q_{1}^{2}} \exp \left(-\frac{P_{1}+c}{Q_{1}}\right) \exp \left(-\frac{P_{2}}{m Q_{2}}\right)<0
$$

Since $P_{1}(t) \geq 0$ and $\mu(t)>0$ for all $t$, then $\lambda(t)<0$, for $t \geq \tau$. So $\lambda(t) \rightarrow-\infty$, as $t \rightarrow \infty$. That means the increasing of the quality would decrease the profit infinitely, which is impossible. So $\lambda(t)>0$, for all $t \geq 0$.

(c) Suppose that the profits for open source and closed source intersect for some value of $k$. Since closed source profit is monotonically increasing in $k$ (Use Envelop Theorem and equation (21), we have $\frac{d V}{d k}=\frac{\partial L}{\partial k}=\lambda N>0$ ) and open source profit is unchanging in $k$, there will be a single point of intersection-a threshold value $\bar{k}$. Suppose that the profits for open source and closed source intersect for some value of $w$. Since closed source profit is monotonically decreasing in $w$ (Use Envelop Theorem and equation (21), we have $\left.\frac{d V}{d w}=\frac{\partial L}{\partial w}=-N^{2}<0\right)$ and open source profit is unchanging in $w$, there will be a single point of intersection - a threshold value $\bar{w}$.

\section{Appendix E: Proof to proposition 5}

We apply the same method as in Appendix 2 to prove the existence of the steady state.

We denote by $\underline{k}=\left(k_{1}, k_{2}\right)=\left(Q_{1}, m\right)$ the vector of the state variables and by $\underline{x}=\left(P_{1}, P_{2}, N\right)$ the vector of control variables, $\underline{q}=\left(q_{1}, q_{2}\right)=(\lambda, \mu)$ are the vector of the adjoint variables or shadow prices. 
Let

$$
\begin{aligned}
& U(\underline{k}, \underline{x})=P_{1} \exp \left(-\frac{P_{1}+c}{Q_{1}}\right) \exp \left(-\frac{P_{2}}{m Q_{2}}\right)+P_{2} \exp \left(-\frac{P_{2}}{m Q_{2}}\right)-w N^{2} \\
& V\left(\underline{k}_{0}\right)=\max _{\underline{x}} \int_{0}^{\infty} e^{-\rho t} U(\underline{k}, \underline{x}) d t
\end{aligned}
$$

Lemma 6: The profit function $U(\underline{k}, \underline{x})$ in closed source problem is concave in $(\underline{k}, \underline{x})$.

Proof: Apply the similar proof as the proof of Lemma 1.

Lemma 7: If $U(\underline{k}, \underline{x})$ is concave in $(\underline{k}, \underline{x}))$, then $V(\cdot)$ is concave.

Proof: By the definition of concavity.

Under the assumption $P_{1}, P_{2}, N>0$

We have

$$
\begin{aligned}
& \dot{k}_{1} \equiv \dot{Q}_{1}=\frac{\partial H}{\partial \lambda}=k N(\underline{k}, \underline{q})-\delta Q_{1} \equiv H_{q_{1}} \\
& \dot{k_{2}} \equiv \dot{m}=a \exp \left(-\frac{P_{1}(\underline{k}, \underline{q})+c}{Q_{1}}\right) \exp \left(-\frac{P_{2}(\underline{k}, \underline{q})}{m Q_{2}}\right)+\exp \left(-\frac{P_{2}(\underline{k}, \underline{q})}{m Q_{2}}\right)-\varepsilon m \equiv H_{q_{2}}
\end{aligned}
$$

So

$$
\begin{aligned}
& H_{q q}=\left[\begin{array}{cc}
\frac{\partial H_{q_{1}}}{\partial \lambda} & \frac{\partial H_{q_{1}}}{\partial \mu} \\
\frac{\partial H_{q_{2}}}{\partial \lambda} & \frac{\partial H_{q_{2}}}{\partial \mu}
\end{array}\right]=
\end{aligned}
$$

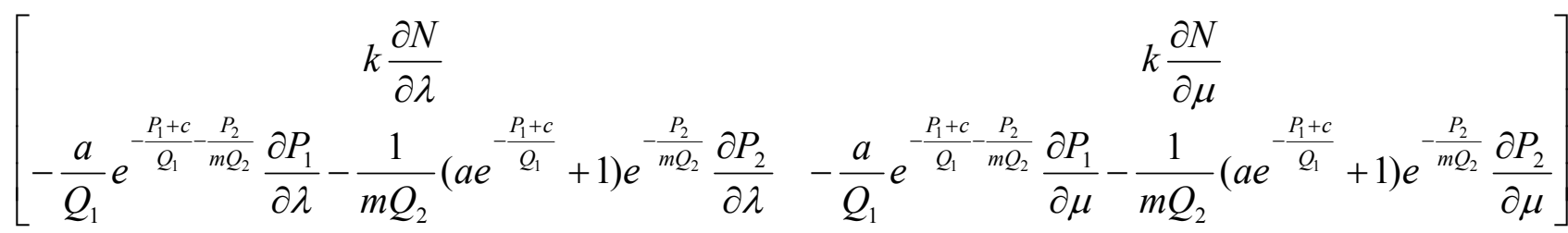$$
H_{q k}=\left[\begin{array}{cc}
\frac{\partial H_{q_{1}}}{\partial Q_{1}} & \frac{\partial H_{q_{1}}}{\partial m} \\
\frac{\partial H_{q_{2}}}{\partial Q_{1}} & \frac{\partial H_{q_{2}}}{\partial m}
\end{array}\right]
$$ 


$$
\begin{aligned}
& =\left[\begin{array}{c}
k \frac{\partial N}{\partial Q_{1}}-\delta \\
\frac{a\left(P_{1}+c\right)}{Q_{1}^{2}} e^{-\frac{P_{1}+c}{Q_{1}}-\frac{P_{2}}{m Q_{2}}}-\frac{a}{Q_{1}} e^{-\frac{P_{1}+c}{Q_{1}}-\frac{P_{2}}{m Q_{2}}} \frac{\partial P_{1}}{\partial Q_{1}}-\frac{1}{m Q_{2}}\left(a e^{-\frac{P_{1}+c}{Q_{1}}}+1\right) e^{-\frac{P_{2}}{m Q_{2}}} \frac{\partial P_{2}}{\partial Q_{1}}
\end{array}\right. \\
& k \frac{\partial N}{\partial m} \\
& \left.\frac{P_{2}}{m^{2} Q_{2}}\left(a e^{-\frac{P_{1}+c}{Q_{1}}}+1\right) e^{-\frac{P_{2}}{m Q_{2}}}-\frac{a}{Q_{1}} e^{-\frac{P_{1}+c}{Q_{1}}-\frac{P_{2}}{m Q_{2}}} \frac{\partial P_{1}}{\partial m}-\frac{1}{m Q_{2}}\left(a e^{-\frac{P_{1}+c}{Q_{1}}}+1\right) e^{-\frac{P_{2}}{m Q_{2}}} \frac{\partial P_{2}}{\partial m}-\varepsilon\right]
\end{aligned}
$$

We need to derive $\frac{\partial N}{\partial \lambda}, \frac{\partial P_{1}}{\partial \lambda}, \frac{\partial P_{2}}{\partial \lambda}, \frac{\partial N}{\partial \mu} \frac{\partial P_{1}}{\partial \mu}, \frac{\partial P_{2}}{\partial \mu}, \frac{\partial N}{\partial Q_{1}}, \frac{\partial P_{1}}{\partial Q_{1}}, \frac{\partial P_{2}}{\partial Q_{1}}, \frac{\partial N}{\partial m}, \frac{\partial P_{1}}{\partial m}, \frac{\partial P_{2}}{\partial m}$

From equation (35) - (37), we have

$$
\begin{aligned}
& f_{1} \equiv P_{1}-Q_{1}+a \mu=0 \\
& f_{2} \equiv P_{2}-m Q_{2}+\mu+\left(P_{1}+a \mu\right) \exp \left(-\frac{P_{1}+c}{Q_{1}}\right)=0 \\
& f_{3} \equiv N-\frac{k \lambda}{2 w}=0
\end{aligned}
$$

Using the implicit function theorem we obtain:

$$
|J|=\left|\begin{array}{lll}
\frac{\partial f_{1}}{\partial P_{1}} & \frac{\partial f_{1}}{\partial P_{2}} & \frac{\partial f_{1}}{\partial N} \\
\frac{\partial f_{2}}{\partial P_{1}} & \frac{\partial f_{2}}{\partial P_{2}} & \frac{\partial f_{2}}{\partial N_{1}} \\
\frac{\partial f_{3}}{\partial P_{1}} & \frac{\partial f_{3}}{\partial P_{2}} & \frac{\partial f_{3}}{\partial N_{1}}
\end{array}\right|=\left|\begin{array}{ccc}
1 & 0 & 0 \\
\left(1-\frac{P_{1}+a \mu}{Q_{1}}\right) e^{-\frac{P_{1}+c}{Q_{1}}} & 1 & 0 \\
0 & 0 & 1
\end{array}\right|=\left|\begin{array}{ccc}
1 & 0 & 0 \\
0 & 1 & 0 \\
0 & 0 & 1
\end{array}\right|=1>0
$$

Since $P_{1}=Q-a \mu$

And

$$
\begin{array}{ll}
\frac{\partial N}{\partial \lambda}=\frac{k}{2 w}>0, & \frac{\partial N}{\partial \mu}=0 \\
\frac{\partial P_{1}}{\partial \lambda}=0, & \frac{\partial P_{1}}{\partial \mu}=-a<0 \\
\frac{\partial P_{2}}{\partial \lambda}=0, & \frac{\partial P_{2}}{\partial \mu}=-\left(a e^{-\frac{P_{1}+c}{Q_{1}}}+1\right)<0
\end{array}
$$




$$
\begin{array}{lll}
\frac{\partial N}{\partial Q_{1}}=0, & \frac{\partial N}{\partial m}=0 \\
\frac{\partial P_{1}}{\partial Q_{1}}=1>0, & \frac{\partial P_{1}}{\partial m}=0 \\
\frac{\partial P_{2}}{\partial Q_{1}}=-\frac{P_{1}+c}{Q_{1}} e^{-\frac{P_{1}+c}{Q_{1}}}<0, & \frac{\partial P_{2}}{\partial m}=Q_{2}>0
\end{array}
$$

We have

$$
H_{q q}=\left[\begin{array}{cc}
\frac{k^{2}}{2 w}>0 & 0 \\
0 & \frac{a^{2}}{Q_{1}} e^{-\frac{P_{1}+c}{Q_{1}}-\frac{P_{2}}{m Q_{2}}}+\frac{1}{m Q_{2}}\left(a e^{-\frac{P_{1}+c}{Q_{1}}}+1\right)^{2} e^{-\frac{P_{2}}{m Q_{2}}}>0
\end{array}\right],
$$

so $H_{q q}$ is symmetric and positive definite. In addition $V$ is concave so $\frac{\partial^{2} V}{\partial \underline{k}^{2}}$ negative semi-definiteness and symmetry follows.

$$
J_{F_{2}}=H_{q q} \frac{\partial^{2} V}{\partial \underline{k}^{2}}+H_{q k}
$$

By lemma 5,

$$
\operatorname{trace}(\mathrm{J})=\operatorname{trace}\left(H_{q q} \frac{\partial^{2} V}{\partial \underline{k}^{2}}\right)+\operatorname{trace}\left(H_{q k}\right) \leq \operatorname{trace}\left(H_{q k}\right)
$$

Compute trace $\left(H_{q k}\right)=-\delta-\varepsilon-\frac{1}{m^{2} Q_{2}}\left(m Q_{2}-P_{2}\right)\left(a e^{-\frac{c}{Q_{1}}}+1\right) e^{-\frac{P_{2}}{m Q_{2}}}<0$

Theorem 3: In the closed source case, if

(a) at the steady state, $\operatorname{trace}\left(H_{q k}\right)<0$

and (b) there is a closed curve $\Gamma$ containing the steady state such that

$$
\underline{\dot{k}}=H_{q}(q(\underline{k}), \underline{k})
$$

'point inward' on $\Gamma$, then, the steady state is locally asymptotically stable (except for hairline cases). 
The condition b) is saying that $\dot{k}_{i}>0$ if $k_{i}$ is small enough and $\dot{k}_{i}<0$ if $k_{i}$ is large enough. In our case, $\dot{Q}_{1}>0$ if $Q_{1}$ is small enough and $\dot{Q}_{1}<0$ if $Q_{1}$ is large enough; and $\dot{m}>0$ if $m$ is small enough and $\dot{m}<0$ if $m$ is large enough.

\section{Appendix F: Proof to proposition 6}

We reduce equation $(31)-(37)$ to two equations.

From (31),

$$
N=\frac{\delta Q_{1}}{k}
$$

Let $(37)=(\mathrm{F} 1)$

$$
\lambda=\frac{2 w \delta Q_{1}}{k^{2}}
$$

Put (35) and (F2) into (33), we get

$$
\begin{aligned}
& \exp \left(-\frac{P_{2}}{m Q_{2}}\right)=\frac{(\rho+\delta) 2 w \delta Q_{1}^{2}}{\left(P_{1}+c\right) k^{2}} \exp \left(\frac{P_{1}+c}{Q_{1}}\right) \\
& P_{2}=-m Q_{2}\left[\ln \frac{(\rho+\delta) 2 w \delta Q_{1}^{2}}{\left(Q_{1}-a \mu+c\right) k^{2}}+\frac{Q_{1}-a \mu+c}{Q_{1}}\right]
\end{aligned}
$$

Let $(36)=(\mathrm{F} 4)$,

$$
\begin{gathered}
m=\frac{\mu+Q_{1} \exp \left(-\frac{Q_{1}-a \mu+c}{Q_{1}}\right)}{\left(\ln \frac{(\rho+\delta) 2 w \delta Q_{1}^{2}}{\left(Q_{1}-a \mu+c\right) k^{2}}+\frac{Q_{1}-a \mu+c}{Q_{1}}+1\right) Q_{2}} \\
-\frac{P_{2}}{m}=\left(\ln \frac{(\rho+\delta) 2 w \delta Q_{1}^{2}}{\left(Q_{1}-a \mu+c\right) k^{2}}+\frac{Q_{1}-a \mu+c}{Q_{1}}\right) Q_{2}
\end{gathered}
$$

Put (36) into (34),

$$
\dot{\mu}=(\rho+\varepsilon) \mu-\frac{P_{2}}{m} \exp \left(-\frac{P_{2}}{m Q_{2}}\right)=0
$$


Put (35), (F3) and (F6) into (F7),

$$
F_{1}\left(Q_{1}, \mu\right)=(\rho+\varepsilon) \mu+Q_{2}\left[\ln \frac{(\rho+\delta) 2 w \delta Q_{1}^{2}}{\left(Q_{1}-a \mu+c\right) k^{2}}+\frac{Q_{1}-a \mu+c}{Q_{1}}\right] \frac{(\rho+\delta) 2 w \delta Q_{1}^{2}}{\left(Q_{1}-a \mu+c\right) k^{2}} \exp \left(\frac{Q_{1}-a \mu+c}{Q_{1}}\right)=0
$$

Put (F3) and (F6) into (32),

$$
\begin{aligned}
F_{2}\left(Q_{1}, \mu\right)= & \left(a \exp \left(-\frac{Q_{1}-a \mu+c}{Q_{1}}\right)+1\right)\left[\ln \frac{(\rho+\delta) 2 w \delta Q_{1}^{2}}{\left(Q_{1}-a \mu+c\right) k^{2}}+\frac{Q_{1}-a \mu+c}{Q_{1}}+1\right] \\
& \frac{(\rho+\delta) 2 w \delta Q_{1}^{2} Q_{2}}{\left(Q_{1}-a \mu+c\right) k^{2}} \exp \left(\frac{Q_{1}-a \mu+c}{Q_{1}}\right)-\varepsilon\left(\mu+Q_{1} \exp \left(-\frac{Q_{1}-a \mu+c}{Q_{1}}\right)\right)=0
\end{aligned}
$$

Given $Q_{1}$, we can calculate the value of $\mu_{1}$, which satisfies $F_{1}\left(Q_{1}, \mu_{1}\right)=0$ and the value of $\mu_{2}$, which satisfies $F_{2}\left(Q_{1}, \mu_{2}\right)=0$, conditioned on $P_{1}\left(Q_{1}, \mu\right)>0$ and $P_{2}\left(Q_{1}, \mu\right)>0$ (At steady state, prices are positive). For the graph below, we plot the points on the range of $Q_{1}$ which meets the positive price condition.

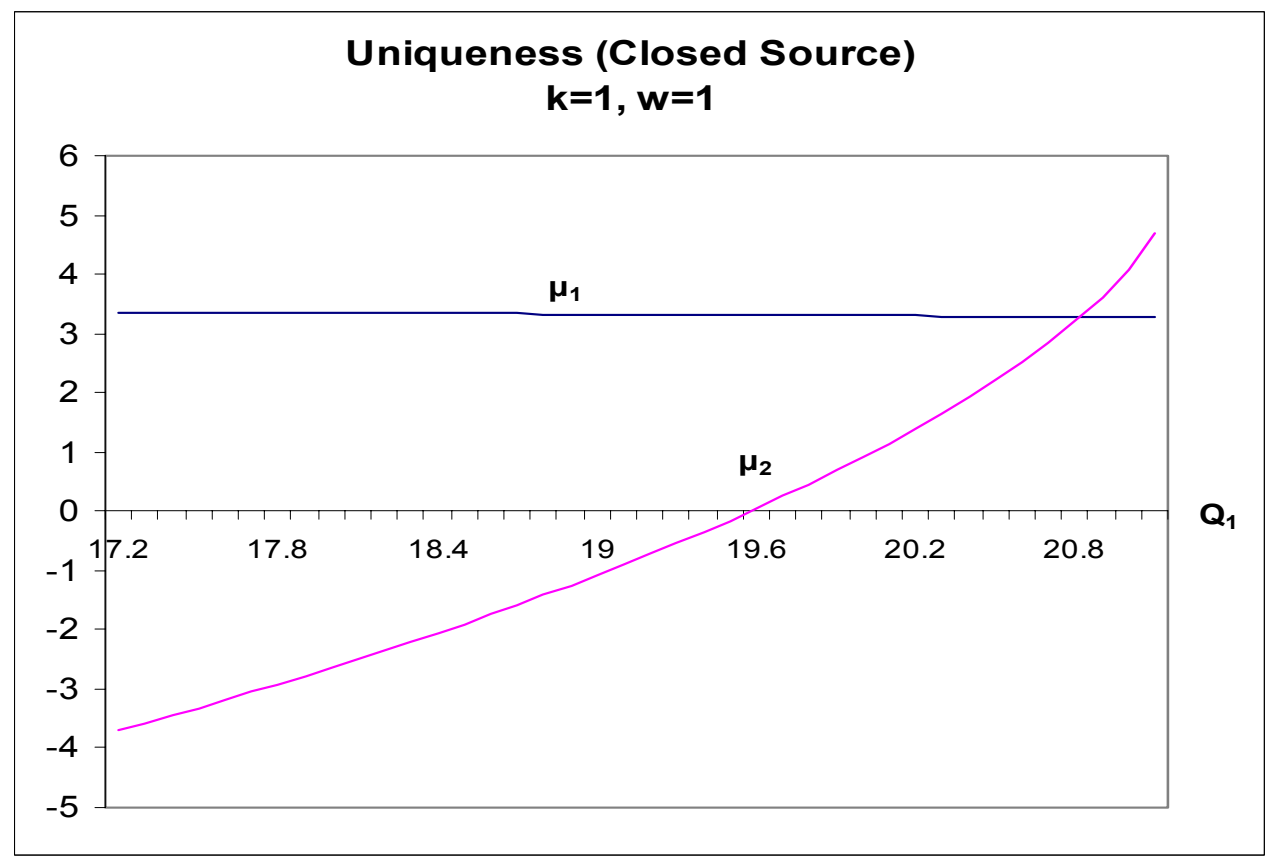

From this graphical illustration, $\mu_{1}$ decreases from a positive number as $Q_{1}$ increases, whereas $\mu_{2}$ increases from a negative number as $Q_{1}$ increases. So there exist unique $\bar{Q}_{1}$ and $\bar{\mu}$ satisfy both $F_{1}\left(\bar{Q}_{1}, \bar{\mu}\right)=0$ and $F_{2}\left(\bar{Q}_{1}, \bar{\mu}\right)=0$. Therefore, $\bar{m}, \bar{P}_{1}, \overline{P_{2}}, \bar{\lambda}, \bar{N}$ are unique. 


\section{Appendix G: Proof to proposition 7}

Condition $N=\frac{k \lambda}{2 w}$ need to be satisfied for all $t \geq 0$.

In the finite horizon without salvage value problem, $\lambda(T)=0$. Hence, $\lambda$ starts positive and declines to zero by time $\mathrm{T}$. So does $N$. Note that $\lambda(T)=0$ means that quality has no salvage value by time T. Hence, it is intuitive that the firm would not wish to spend money to hire developers towards the end of the life of the product.

In the infinite horizon problem, the existence and the uniqueness assure that $N$ is constant after a sufficiently long period of time. 